\title{
Ciberacoso según caracteristicas sociodemográficas y académicas en estudiantes universitarios
}

\section{Cyberbullying according to sociodemographic and academic characteristics among university students}

\author{
Dra. Inmaculada MÉNDEZ. Profesora Ayudante Doctor. Universidad de Murcia (inmamendez@um.es). \\ Dra. Cecilia RUIZ ESTEBAN. Profesora Titular. Universidad de Murcia (cruiz@um.es). \\ Dr. Juan Pedro MARTíNEZ. Profesor Asociado. Universidad de Murcia (juanpedromartinezramon@um.es). \\ Dra. Fuensanta CEREZO. Profesora Honoraria. Universidad de Murcia (fcerezo@um.es).
}

\section{Resumen:}

Existe evidencia de la aparición del acoso escolar en el ámbito universitario tanto de manera presencial como a través de medios tecnológicos. El objetivo del estudio fue analizar las diferencias entre los roles del ciberacoso (objeto, agente y observador) en función de variables sociodemográficas y académicas (sexo, edad, procedencia, nivel de estudios y Facultad). Los participantes del estudio fueron 765 jóvenes estudiantes $(72.9 \%$ mujeres) de una universidad del sureste español (83.7\% estudios de Grado, el 15.2\% de Máster y el $1.1 \%$ otros estudios) pertenecientes a diferentes Facultades (Humanidades, Ciencias Sociales y de la Educación, Ciencias de la Salud y Ciencias). El instrumento utilizado fue el Cuestionario sobre Acoso entre Estudiantes
Universitarios. Los resultados del estudio determinaron que las mujeres, los menores de 20 años, los estudiantes de Grado, los estudiantes de Humanidades, Ciencias Sociales y Ciencias de la Salud obtuvieron valores más altos en las subescalas evaluadas. Entre los roles de los implicados destacaban los observadores seguidos de agresores y de víctimas. $\mathrm{El}$ análisis de regresión determinó la relación entre el hecho de haber sido objeto de ciberacoso, agente de ciberacoso y observador de ciberacoso. El estudio permitirá centrarse en aquellas variables sociodemográficas que resultaron ser significativas, así como la relación entre los roles del ciberacoso de cara a programas de prevención e intervención en cada uno de sus roles. El contexto universitario debe asumir la importancia de promover la

Fecha de recepción de la versión definitiva de este artículo: 11-02-2019.

Cómo citar este artículo: Méndez, I., Ruiz Esteban, C., Martínez, J. P. y Cerezo, F. (2019). Ciberacoso según características sociodemográficas y académicas en estudiantes universitarios | Cyberbullying according to sociodemographic and academic characteristics among university students. Revista Española de Pedagogía, 77 (273), 261-276. doi: https:// doi.org/10.22550/REP77-2-2019-06 
convivencia y el bienestar universitario. Asimismo, es relevante la implicación de toda la comunidad educativa.

Descriptores: cibernética, universidad, facultad universitaria, educación, ciberacoso.

\section{Abstract:}

There is evidence of the existence of both bullying and cyberbullying in the university environment. The aim of this study is to analyse the differences between cyberbullying roles (target, perpetrator, and bystander) according to sociodemographic and academic variables (sex, age, origin, level of studies, and faculty). The participants were 765 young students ( $72.9 \%$ women) from a university of the southeast of Spain $(83.7 \%$ undergraduate degree, $15.2 \%$ Master's degree, and $1.1 \%$ other studies) from different faculties. The Questionnaire on Harassment among University Students tool was used.

\section{Introducción}

Durante la etapa educativa pueden aparecer problemas de intimidación o de hostigamiento entre los iguales (Ortega-Ruiz, 2015) sobre todo en las minorías étnicas-culturales, sexuales, etc. (Llorent, Ortega-Ruiz y Zych, 2016; Walker, 2015). Dichas situaciones se caracterizan por poseer un carácter intencional, ser reiteradas en el tiempo y llevadas a cabo por un individuo 0 un grupo contra una víctima que no es capaz de defenderse, existiendo por tanto, un desequilibrio de poder (Olweus, 2013).
The results of the study determined that women, people aged under 20, undergraduate degree students, and Humanities, Social Sciences, and Health Sciences students obtained higher values in the sub-scales evaluated. Among the roles of those involved, bystanders stood out, followed by perpetrators and victims. Regression analysis showed a relationship between being the target, perpetrator, and/or bystander of cyberbullying. The study will make it possible to focus on those sociodemographic variables that turned out to be significant as well as the relationship between the cyberbullying roles in the face of prevention and intervention programs for each of the roles. The university context must assume the importance of promoting coexistence and university welfare. The involvement of the entire educational community is also relevant.

Keywords: cybernetics, universities, university faculty, education, cyberbullying.
El ciberacoso consiste en utilizar las TIC - Tecnologías de la Información y de la Comunicación - para ejercer acoso entre iguales, sobre todo a través de internet o del uso del móvil. Las vías utilizadas suelen ser mensajes de texto, acoso a través de llamadas, grabaciones de agresiones 0 vejaciones que se difunden, redes sociales, etc. (Garaigordobil, 2015), siendo estas situaciones repetidas en el tiempo con intencionalidad y desequilibrio de poder (Zych, Ortega-Ruiz y Marín-López, 2016). Generalmente existen tres grupos de actores implicados directamente en esta proble- 
mática: víctima u objeto, agente y observador tanto de forma directa como a través del ciberacoso.

Se tiende a encubrir el ciberacoso (Hernández y Solano, 2007), la ocultación de identidad del agresor facilita su impunidad y aumenta la indefensión de la víctima. Las agresiones electrónicas se difunden rápidamente a un gran número de personas pudiendo ser reenviadas a su vez (Buelga, Cava y Musitu, 2010).

El mundo virtual ofrece posibilidades, pero también riesgos como, por ejemplo, la ciberadicción 0 el ciberacoso (Arnaiz, Cerezo, Giménez y Maquilón, 2016; Del Rey, Casas y Ortega-Ruiz, 2012; Myers y Cowie, 2017). Del Rey et al., (2012) aluden que el abuso de las nuevas tecnologías es un riesgo que puede afectar a la calidad de vida de los estudiantes, debido a la situación de dependencia que genera. Los estudios han demostrado que el ciberacoso puede generar consecuencias legales y psicológicas muy negativas para los estudiantes implicados (Álvarez-García, Barreiro-Collazo, Núñez y Dobarro, 2016). Ortega-Barón, Buelga, Cava y Torralba (2017) evidenciaron que los estudiantes que habían sido ciberagresores de forma severa y los que lo habían hecho de forma ocasional, habían transgredido las normas, es decir, que a su vez se habían implicado en otros comportamientos violentos.

Entre los factores de protección cabe destacar que la autoestima es un factor de protección de la cibervictimización ocasional frente al hecho de tener móvil propio, jugar on-line y la frecuencia de uso de internet los días de la semana. La cibervictimización tanto ocasional como de forma severa se da sobre todo en las chicas y con una media de edad de 14 años (Álvarez-García, Núñez, Dobarro y Rodríguez, 2016). Asimismo, Arnaiz et al., (2016) aluden que la supervisión familiar actúa como factor de protección.

La simultaneidad del acoso tradicional y del ciberacoso hace suponer que a partir de la implicación en el acoso tradicional se puede predecir la implicación en el ciberacoso (cibervictimización y ciberagresión) (Del Rey, Elipe y Ortega-Ruiz, 2012; Yubero, Navarro, Elche, Larrañaga y Ovejero, 2017). Los datos del informe elaborado por Save the Children en España (Sastre, 2016) sobre acoso y ciberacoso revelaron que $9.3 \%$ de los estudiantes había sido víctima de acoso escolar y el $6.9 \%$ víctima de ciberacoso. Entre las formas de manifestación, cabe destacar que el insulto fue la forma de manifestación más frecuente (seis de cada 10 estudiantes habían insultado y más de dos de cada 10 lo habían sufrido frecuentemente), seguido de otras formas como rumores, robo, amenazas, maltrato físico o exclusión. Asimismo, el 5.4\% había sufrido ciberacoso y el $3.3 \%$ había realizado ciberacoso. Lo que evidencia que la prevalencia del ciberacoso ha ido en aumento (Álvarez-García et al., 2016; Garaigordobil, 2011, 2015).

Por lo tanto, se trata de un problema social que transciende las barreras de los contextos educativos de la educación primaria y secundaria, afectando a otros tipos de población como en los escenarios universitarios teniendo los mismos efectos devastadores (Crosslin y Golman, 2014; García-Peña, Moncada y Quintero, 2013; Torres-Mora, 2010; Walker, 2015). 
Hasta hace unos años no se había tenido en cuenta el riesgo de la población universitaria puesto que se le atribuía cierta madurez psicosocial, lo que hacía que se minimizasen los problemas de relaciones entre los universitarios. Se ha de tener en cuenta que la intimidación entre jóvenes universitarios no se trata únicamente de una problemática social sino que supone un problema de salud pública. De lo anterior se desprende que el acoso escolar no es un fenómeno exclusivo del ámbito escolar de la educación primaria y secundaria sino que se presenta como un problema de relaciones entre iguales, que puede ocurrir en lugares académicos, lugares universitarios, etc. y que dicho abuso de poder se presenta incluso a través de los medios tecnológicos (ciberacoso). Se trata de un problema serio que genera consecuencias negativas en el desarrollo de los estudiantes universitarios (Cassidy, Faucher y Jackson, 2017; Crosslin et al., 2014; García-Peña et al., 2013; Walker, 2015).

En el ambiente universitario existen una serie de factores generadores de estrés, ansiedad, sintomatología depresiva... Uno de esos factores es la intimidación 0 acoso entre escolares (García-Peña et al., 2013). Entre los factores que contribuyen a la aparición de intimidación en el ambiente universitario cabe destacar las diferencias en las capacidades académicas, grupo de iguales, estudiantes con necesidades (McDougal, 1999).

Entre los universitarios se ha evidenciado la existencia de menos agresiones físicas en comparación con las ocurridas en otras etapas educativas, mientras que son mayo- res otros tipos de hostilidad tales como el maltrato verbal (burlas, insultos, etc.), la exclusión social a través de la marginación, denigración, abusos en los trabajos en equipo, tipificación por rasgos físicos y posición económica, entre otros (Hoyos, Romero, Valega y Molinares, 2009; López, 2017; Paredes, Sanabria-Ferrand, González-Quevedo y Moreno Rehalpe, 2010; Torres-Mora, 2010; Trujillo y Romero-Acosta, 2016). Entre los roles de los implicados destacan los observadores seguido de agresores y de víctimas (Hoyos et al., 2009; Paredes et al., 2010; Trujillo et al., 2016). Tanto hombres como mujeres son actores de las distintas formas de maltrato (Anguiano-Carrasco y Vigil-Colet, 2011; Faucher, Jackson y Cassidy, 2014; Hoyos et al., 2009; Kokkinos, Antoniadou y Markos, 2014). Concretamente, los hombres suelen aparecer más implicados en los roles de víctimas frente a las mujeres, aunque no en todas las manifestaciones, mientras que ellas aparecían como observadoras. En cuanto a los agresores, las mujeres se mostraban como agresoras en las diferentes modalidades (ignorar, hablar mal del otro, etc.) frente a los varones (poner apodos, insultar, etc.) (Hoyos et al., 2009; Hoyos, Llanos y Valega, 2012). En lo que respecta a la titulación, Molero, Gázquez, Pérez-Fuentes y Soler (2014) no encontraron diferencias para el tipo de titulación analizada (Grado en Educación Primaria, Grado en Educación Infantil y Grado en Psicología) al igual que Paredes et al., (2010) que analizaron las situaciones de acoso en las Facultades de Medicina de Colombia. En cuanto a la edad, entre los 17 y 20 años es donde se evidencia más presencia de maltrato en más manifestaciones (Hoyos et al., 2009; Hoyos 
et al., 2012) lo que parece deberse a la dificultad para tener dinámicas de los grupos y a la participación en los mismos de cada uno de los estudiantes.

Por este motivo, el objetivo del estudio fue analizar las diferencias en función de variables sociodemográficas y académicas (sexo, edad, procedencia, nivel de estudios y facultad) entre los roles del ciberacoso (objeto de ciberacoso, ciberacosador como agente y observador de ciberagresiones).

\section{Método}

\subsection{Participantes}

Los participantes del estudio fueron 765 estudiantes de una universidad del sureste español, con edades comprendidas entre menos de 20 años y más de 30 años, siendo el $72.9 \%$ mujeres (véase Tabla 1 ). El 93.5\% de los estudiantes eran de procedencia española. En cuanto al tipo de estudios, el $83.7 \%$ cursaban estudios de Grado, el $15.2 \%$ de Máster y el resto otros estudios. En lo referente a la facultad a la cual estaban adscritos los estudios que realizaban, la distribución fue la siguiente: el 14\% Humanidades (Bellas Artes, Geografía, Historia, Lengua, Lenguas Clásicas, Filosofía y otras), 45.6\% Ciencias Sociales y de la Educación (Derecho, Económicas, Trabajo social, Educación y otras); el $30.2 \%$ Ciencias de la Salud (Medicina, Enfermería, Psicología, Óptica, Fisioterapia y otras) y el $10.2 \%$ Ciencias (Física, Química, Biología, Informática, Ingenierías y otras).

TABLA 1. Distribución de los estudiantes según la edad y el sexo.

\begin{tabular}{|l|r|r|}
\hline \multicolumn{1}{|c|}{ Edad } & Hombre & Mujer \\
\hline Menos de 20 años & $41(19.8 \%)$ & $182(32.8 \%)$ \\
\hline Entre 20-24 años & $125(60.4 \%)$ & $276(49.7 \%)$ \\
\hline Entre 25-29 años & $28(13.5 \%)$ & $58(10.5 \%)$ \\
\hline 30 años o más & $13(6.3 \%)$ & $39(7 \%)$ \\
\hline
\end{tabular}

Fuente: Elaboración propia.

\subsection{Instrumentos}

El instrumento utilizado fue el Cuestionario sobre Acoso entre Estudiantes Universitarios (QAEU) de Cerezo, Martín, Martínez, Méndez y Ruiz (2016) validado por Martínez, Méndez, Ruiz y Cerezo (inédito).

El instrumento consta de 135 ítems. En una primera parte mide variables sociodemográficas y académicas tales como: edad (menos de 20 años, entre 20-24 años, entre
25-29 años y 30 o más años), sexo (hombre/mujer), facultad donde cursaba los estudios (Humanidades, Ciencias Sociales y de la Educación, Ciencias de la Salud y Ciencias), tipo de estudios (Grado, Máster u otro tipo), curso ( $1^{0}, 2^{\underline{0}}, 3^{\circ}$ o $\left.4^{\circ}\right)$ y país de procedencia (español u otro). A continuación, el instrumento mide tres escalas:
a) Como objeto de la agresión.
b) Como agente de las agresiones.
c) Como observador de las agresiones. 
Cada una de ellas mide agresión directa (bullying) así como ciberacoso (ciberbullying):

a) Como objeto de la agresión: consiste en 49 ítems distribuidos entre la agresión directa (tipo, quién, lugar, tiempo y razón) y ciberacoso (tipo, forma o medio, momento, razón, informante y apoyo), se compone de subescalas de agresión directa (AD) (p. ej., he sufrido agresión física) y haber sido objeto del ciberacoso (CA) (p. ej.; en ocasiones he recibido ciberacoso a través de redes sociales).

b) Como agente de agresión: tiene 35 ítems distribuidos entre agresión directa 0 acoso (tipo, objeto o dirección, momento y causa) y ciberacoso (acto, camino, objeto y motivos), y está compuesto

c) Como observador de la agresión: consta de 45 elementos distribuidos entre la observación de la agresión directa 0 el acoso (tipo, objeto, momento y motivos) y el ciberacoso (tipo, lugar, exclusión, causas, informado y ayuda) formado por las subescalas de observador de agresiones directas (OB) (por ejemplo, «en alguna ocasión, he visto agredir a otro estudiante, las agresiones fueron físicas») y observa- dor del acoso cibernético (OBC) (por ejemplo, «en ocasiones he observado el acoso cibernético» —agresión a otros a través de llamadas móviles 0 por Internet).

Dichas subescalas poseen valores Alpha de Cronbach adecuados: como objeto de las agresiones .95; como agente de las agresiones .94 y como observador de las agresiones .96. Para el presente estudio se seleccionaron las subescalas relativas al ciberacoso (CA, CAR y OBC).

\subsection{Procedimiento}

La selección de los participantes se realizó atendiendo a la facultad en la cual estaban matriculados en el curso académico 2015/2016, asegurando la representatividad de cada grupo en las 20 facultades de la universidad objeto de estudio (Humanidades, Ciencias Sociales y de la Educación, Ciencias de la Salud y Ciencias) con un nivel de confianza del $95 \%$. Fue necesario solicitar el permiso y la colaboración de los docentes encargados de los estudios de Grado, Máster u otros seleccionados para que fuese posible administrar los cuestionarios de forma presencial o de forma online a través del aula virtual (28.48\%) de la asignatura. La administración del cuestionario tuvo una duración de entre 15 a 20 minutos. Durante la cumplimentación del instrumento se aseguró la confidencialidad y el anonimato de los datos. El protocolo fue aprobado por el Comité de Ética para Investigaciones Clínicas de la Universidad de Murcia. El estudio se realizó de acuerdo con las directrices aprobadas y la Declaración de Helsinki. 


\subsection{Análisis de datos}

Con la finalidad de analizar los roles del ciberacoso según las características sociodemográficas se realizó una diferencia de medias ( $t$ de Student) para muestras independientes para determinar si existían diferencias por sexo y según la procedencia en cada una de las subescalas y el estadístico $d$ de Cohen (1988) para el tamaño del efecto. Para analizar las diferencias de medias según el nivel de estudios, la facultad en la cual estaban cursando los estudios, así como la edad (en rangos) se utilizó un análisis de Varianza (ANOVA) para cada una de las subescalas. Se utilizaron comparaciones post-hoc mediante la prueba de Bonferroni. Asimismo, se utilizó la prueba de Brown-Forsythe cuando no pudo ser admitida la igualdad de varianzas a través de la prueba de Levene. Se utilizaron análisis de regresión jerárquicos para contrastar la potencia predictiva de los grupos de variables independientes (variables sociodemográficas como la edad, el sexo, facultad, nivel de estudios y procedencia) con respecto a la variable dependiente bajo estudio (CA, CAR, OBC) así como las relaciones entre estas a través del método introducir. Los análisis se realizaron con el SPSS v.21.

\subsection{Resultados}

El $14.4 \%$ de los estudiantes había sido objeto de ciberacoso, el 7.3\% había realizado ciberacoso y el $17.4 \%$ había observado ciberacoso.

La Tabla 2 muestra las diferencias de sexo en las subescalas del QAEU. La prueba $t$ de Student mostró diferencias de medias significativas en las subescalas
$\mathrm{CA}, \mathrm{CAR}$ y $\mathrm{OBC}$ con valores superiores en las mujeres con un tamaño del efecto bajo $(d=-.20)$.

En segundo lugar, la Tabla 3 muestra las diferencias por rangos de edad en las subescalas del QAEU en la prueba ANOVA de un factor. Las pruebas post-hoc revelaron que existían diferencias de medias entre los estudiantes de menos de 20 años y los estudiantes con edades comprendidas entre los 20-24 años en las tres subescalas. En la subescala CAR existían diferencias de medias entre los estudiantes de 20-24 años y los estudiantes de 30 años 0 más, siendo superior en estos últimos.

La Tabla 4 muestra las diferencias por procedencia en las subescalas del QAEU sub-scales. La prueba $t$ de Student no mostró diferencias de medias significativas en las subescalas CA, CAR y OBC.

La Tabla 5 muestra los resultados del estudio según el nivel de estudios. Las pruebas post-hoc indicaban que existían diferencias de medias significativas entre los estudiantes de Grado y los estudiantes de Máster en las tres subescalas (CA, CAR y OBC) siendo inferior en estos. Asimismo, en la subescala CA existían diferencias de medias entre los estudiantes de Máster y los que realizaban otro tipo de formación, siendo superior en estos últimos.

Finalmente, la Tabla 6 muestra los resultados según la facultad a la que pertenecían los estudios en los cuales estaban matriculados los estudiantes. Las pruebas post-hoc indicaban que existían diferen- 
cias de medias significativas entre los estudiantes de Ciencias (obtuvieron valores más bajos) y los estudiantes de Humanidades, Ciencias Sociales y los estudiantes de Ciencias de la Salud en las subescalas CA, CAR y OBC. Asimismo, en la subescala OBC existían diferencias de medias entre los estudiantes de Ciencias Sociales y Ciencias de la Salud, siendo superior en el primero.

Finalmente, el análisis de regresión utilizando como criterio CA y como variables predictoras las relativas a variables sociodemográficas y académicas (edad, sexo, facultad, nivel de estudios y procedencia) así como CAR y OBC, explicó el $65.8 \%$ de la varianza. Los coeficientes de regresión estandarizados Beta indicaron que, de todas las variables predictoras introducidas, resultaron ser significativas el nivel de estudios (Beta $=-.062 ; t=-2.606 ; \mathrm{p}=.009)$; $\mathrm{CAR}($ Beta $=.474 ; t=14.458 ; \mathrm{p}=.000) \mathrm{y}$ OBC $($ Beta $=.379 ; t=11.615 ; \mathrm{p}=.000)$. Asimismo, el análisis de regresión utili- zando como criterio CAR y como variables predictoras las relativas a variables sociodemográficas y académicas (edad, sexo, facultad, nivel de estudios y procedencia) así como CA y OBC, explicó el $66.6 \%$ de la varianza. Los coeficientes de regresión estandarizados Beta indicaron que de todas las variables predictoras introducidas, resultaron significativas las siguientes: facultad (Beta $=-.048 ; t=-2.163$; $\mathrm{p}=.031), \quad$ CA $($ Beta $=.459 ; t=14.458$; $\mathrm{p}=.000)$ OBC $($ Beta $=.409 ; \mathrm{t}=12.974$; $\mathrm{p}=.000$ ). Del mismo modo, el análisis de regresión utilizando como criterio $\mathrm{OBC}$ y como variables predictoras las relativas a variables sociodemográficas y académicas (edad, sexo, facultad, nivel de estudios y procedencia) así como CA y CAR, explicó el 63.6\% de la varianza. Los coeficientes de regresión estandarizados Beta indicaron que de todas las variables predictoras introducidas, resultaron significativas las siguientes: CA (Beta $=.401 ; t=11.615$; $\mathrm{p}=.000)$ y CAR $($ Beta $=.447 ; t=12.974$; $\mathrm{p}=.000)$.

TABLA 2. Subescalas de ciberacoso de QAEU según el sexo.

\begin{tabular}{|l|c|c|c|c|}
\hline \multirow{2}{*}{ SUBESCALA QAEU } & \multicolumn{2}{|c|}{ SEXO } & \multicolumn{2}{|c|}{} \\
\cline { 2 - 5 } & VARONES & MUJERES & \multicolumn{1}{|c|}{} \\
\cline { 2 - 5 } CA & $M(D T)$ & $M(D T)$ & $t$ & $.016^{*}$ \\
\hline CAR & $12.24(14.81)$ & $15.28(15.62)$ & -2.243 & $.019^{*}$ \\
\hline OBC & $5.9(8.22)$ & $7.6(9.2)$ & -2.349 & $.013^{*}$ \\
\hline
\end{tabular}

Nota: $\mathrm{CA}=$ ciberacoso como objeto, $\mathrm{CAR}=$ ciberacoso como agente, $\mathrm{OBC}=$ observador de ciberagresiones; n.s. no significativo.

Fuente: Elaboración propia. 
TABLA 3. Subescalas de ciberacoso de QAEU por edad.

\begin{tabular}{|c|c|c|c|}
\hline \multirow{2}{*}{ SUBESCALA QAEU } & \multirow{2}{*}{$\begin{array}{c}\text { EDAD } \\
F\end{array}$} & \multirow[b]{2}{*}{ Diferencias de medias } & \multirow[b]{2}{*}{$p$} \\
\hline & & & \\
\hline CA & $\begin{array}{l}\text { Brown-Forsythe } \\
F(3 ; 30.423)=3.633 \\
p=.013\end{array}$ & $\begin{array}{l}\text { Menos } 20 \text { años-20-24 años }=3.60 \\
\text { Menos } 20 \text { años-25-29 años }=3.40 \\
\text { Menos } 20 \text { años-30 o más }=-1.35 \\
20-24 \text { años-25-29 años }=-.20 \\
20-24 \text { años-30 o más }=-4.95 \\
25-29 \text { años-30 o más }=-4.75\end{array}$ & $\begin{array}{l}.031^{*} \\
\text { n.s. } \\
\text { n.s. } \\
\text { n.s. } \\
\text { n.s. } \\
\text { n.s. }\end{array}$ \\
\hline CAR & $\begin{array}{l}\text { Brown-Forsythe } \\
F(3 ; 280.899)=6.856 \\
p=.000\end{array}$ & $\begin{array}{l}\text { Menos } 20 \text { años-20-24 años }=3.06 \\
\text { Menos } 20 \text { años-25-29 años }=2.12 \\
\text { Menos } 20 \text { años-30 o más }=-.77 \\
20-24 \text { años-25-29 años }=-.94 \\
20-24 \text { años-30 o más }=-3.83 \\
25-29 \text { años-30 o más }=-2.89\end{array}$ & $\begin{array}{l}.000 \\
\text { n.s. } \\
\text { n.s. } \\
\text { n.s. } \\
.019^{*} \\
\text { n.s. }\end{array}$ \\
\hline OBC & $\begin{array}{l}\text { Brown-Forsythe } \\
F(3 ; 322.231)=5.148 \\
p=.002\end{array}$ & $\begin{array}{l}\text { Menos } 20 \text { años- } 20-24 \text { años }=5.52 \\
\text { Menos } 20 \text { años-25-29 años }=3.44 \\
\text { Menos } 20 \text { años-30 o más }=1.49 \\
20-24 \text { años-25-29 años }=-2.08 \\
20-24 \text { años-30 o más }=-4.04 \\
25-29 \text { años-30 o más }=-1.95\end{array}$ & $\begin{array}{l}.001^{*} \\
\text { n.s. } \\
\text { n.s. } \\
\text { n.s. } \\
\text { n.s. } \\
\text { n.s. }\end{array}$ \\
\hline
\end{tabular}

Nota: $\mathrm{CA}=$ Ciberacoso como objeto $\mathrm{CAR}=$ Ciberacoso como agente; $\mathrm{OBC}=$ Observador de ciberagresiones; n.s. no significativo.

Fuente: Elaboración propia.

TABLA 4. Diferencias por procedencia en las subescalas de ciberacoso de QAEU.

\begin{tabular}{|l|c|c|c|c|}
\hline \multirow{2}{*}{ SUBESCALA QAEU } & \multicolumn{2}{|c|}{ PROCEDENCIA } & \multicolumn{2}{|c|}{} \\
\cline { 2 - 5 } & ESPANOL & OTROS & \multicolumn{2}{|c|}{} \\
\cline { 2 - 5 } CA & $M(D T)$ & $M(D T)$ & $t$ & n.s. \\
\hline \multirow{2}{*}{ CAR } & $14.46(15.42)$ & $14.56(16.35)$ & -.045 & n.s. \\
\hline \multirow{2}{*}{ OBC } & $7.17(8.92)$ & $7.14(9.37)$ & -.020 & n.s. \\
\hline
\end{tabular}

Nota: $\mathrm{CA}=$ Ciberacoso como objeto; $\mathrm{CAR}=$ Ciberacoso como agente; $\mathrm{OBC}=$ Observador de ciberagresiones; n.s. no significativo.

Fuente: Elaboración propia. 
TABLA 5. Diferencias por el nivel de estudios en las subescalas de ciberacoso de QAEU.

\begin{tabular}{|c|c|c|c|}
\hline \multirow{2}{*}{ SUBESCALA QAEU } & NIVEL DE ESTUDIOS & & \\
\hline & $F$ & Diferencias de medias & $p$ \\
\hline CA & $\begin{array}{l}\text { Brown-Forsythe } \\
F(2 ; 24.544)=21.645 \\
p=.000\end{array}$ & $\begin{array}{l}\text { Grado-Máster }=8.37 \\
\text { Grado-Otros }=-8.67 \\
\text { Máster-Otros }=-17.04\end{array}$ & $\begin{array}{l}.000 * \\
\text { n.s. } \\
.07 *\end{array}$ \\
\hline CAR & $\begin{array}{l}\text { Brown-Forsythe } \\
F(2 ; 22.451)=9.085 \\
p=.001\end{array}$ & $\begin{array}{l}\text { Grado- Máster }=3.45 \\
\text { Grado-Otros }=-2.30 \\
\text { Máster-Otros }=5.76\end{array}$ & $\begin{array}{l}.000 * \\
\text { n.s. } \\
.07 *\end{array}$ \\
\hline OBC & $\begin{array}{l}\text { Brown-Forsythe } \\
F(2 ; 17.985)=9.764 \\
p=.001\end{array}$ & $\begin{array}{l}\text { Máster-Otros }=5.76 \\
\text { Grado-Máster }=7.14 \\
\text { Grado-Otros }=-5.80 \\
\text { Máster-Otros }=-12.93\end{array}$ & $\begin{array}{l}\text { n.s. } \\
.000^{*} \\
\text { n.s. } \\
\text { n.s. }\end{array}$ \\
\hline
\end{tabular}

Nota: $\mathrm{CA}=$ Ciberacoso como objeto $\mathrm{CAR}=$ Ciberacoso como agente $\mathrm{OBC}=$ Observador de ciberagresiones; n.s. no significativo.

Fuente: Elaboración propia.

TABLA 6. Diferencias por Facultad en las subescalas de QAEU.

\begin{tabular}{|c|c|c|c|}
\hline \multirow{2}{*}{ SUBESCALA QAEU } & \multirow{2}{*}{$\begin{array}{c}\text { FACULTAD } \\
F \\
\end{array}$} & \multirow{2}{*}{ Diferencias de medias } & \multirow[b]{2}{*}{$p$} \\
\hline & & & \\
\hline CA & $\begin{array}{l}\text { Brown-Forsythe } \\
F(3 ; 508.722)=8.240 \\
p=.000\end{array}$ & $\begin{array}{l}\text { HDs - C. Sociales }=1.81 \\
\text { HDs - C. Salud }=-.424 \\
\text { HDs - Ciencias }=8.85 \\
\text { C. Sociales - C. Salud }=-2.23 \\
\text { C. Sociales - Ciencias }=7.04 \\
\text { C. Salud - Ciencias }=9.27\end{array}$ & $\begin{array}{l}\text { n.s. } \\
\text { n.s. } \\
.001^{*} \\
\text { n.s. } \\
.002^{*} \\
.000^{*}\end{array}$ \\
\hline CAR & $\begin{array}{l}\text { Brown-Forsythe } \\
F(3 ; 523.363)=12.136 \\
p=.000\end{array}$ & $\begin{array}{l}\text { HDs - C. Sociales }=2.1 \\
\text { HDs - C. Salud }=.56 \\
\text { HDs - Ciencias }=6.5 \\
\text { C. Sociales - C. Salud }=-1.54 \\
\text { C. Sociales - Ciencias }=4.41 \\
\text { C. } \text { Salud - Ciencias }=5.95\end{array}$ & $\begin{array}{l}. \mathrm{n} . \mathrm{s} . \\
\mathrm{n} . \mathrm{s} . \\
.000 * \\
\text { n.s. } \\
.000^{*} \\
.000^{*}\end{array}$ \\
\hline OBC & $\begin{array}{l}\text { Brown-Forsythe } \\
F(3 ; 507.807)=10.126 \\
p=.000\end{array}$ & $\begin{array}{l}\text { HDs - C. Sociales }=2.89 \\
\text { HDs - C. Salud }=-1.13 \\
\text { HDs - Ciencias }=10.05 \\
\text { C. Sociales - C. Salud }=-4.02 \\
\text { C. Sociales - Ciencias }=7.17 \\
\text { C. } \text { Salud - Ciencias }=11.19\end{array}$ & $\begin{array}{l}\text { n.s. } \\
\text { n.s. } \\
.000^{*} \\
.031^{*} \\
.004^{*} \\
.000^{*}\end{array}$ \\
\hline
\end{tabular}

Nota: $\mathrm{CA}=$ Ciberacoso como objeto; $\mathrm{CAR}=$ Ciberacoso como agente; $\mathrm{OBC}=$ Observador de ciberagresiones; n.s. no significativo.

Fuente: Elaboración propia. 


\section{Discusión}

Los estudiantes universitarios, al igual que en otras etapas educativas, son igualmente vulnerables a la problemática del acoso entre escolares (Crosslin et al., 2014; García-Peña et al., 2013). Entre los roles de los implicados destacaban los observadores seguido de agresores y de víctimas (Hoyos, et al., 2009; Paredes et al., 2010; Trujillo et al., 2016). Ello hace suponer que las situaciones de agresión son encubiertas entre los universitarios, siendo difíciles de detectar. Debido a que en el ámbito universitario no suelen relacionarse todos los estudiantes del Grado, Máster o de otros cursos, sino que las relaciones se reducen a un grupo de iguales, la identificación de dichas situaciones de agresividad solo puede darse entre los estudiantes más cercanos (Hoyos et al., 2009). La percepción de que la intimidación es algo legitimado dentro del grupo social de los estudiantes, hace que se valore de forma positiva (Paredes et al., 2010). Por lo tanto, se tiende a encubrir el ciberacoso (Hernández et al., 2007) ya que la pasividad de la víctima y del observador supone el sometimiento a un poder que le impide el derecho a la libertad (Torres-Mora, 2010). El ciberacoso puede generar consecuencias legales y psicológicas muy negativas para los estudiantes implicados (Álvarez-García, Barreiro-Collazo et al., 2016; Cassidy et al., 2017; Crosslin et al., 2014; Walker, 2015; Yubero et al., 2017).

En nuestro estudio tanto hombres como mujeres fueron actores de las distintas formas de maltrato (Anguiano-Carrasco et al., 2011; Faucher et al., 2014; Hoyos et al., 2009; Kokkinos et al., 2014). Las mujeres obtuvieron valores más altos en todas las subescalas (como agente, objeto y observador) lo que está en concordancia con estudios anteriores (Hoyos et al., 2009; Hoyos, Llanos y Valega, 2012). En lo que respecta a la edad, cabe destacar que los resultados mostraron que en todas las subescalas los menores de 20 años obtuvieron valores más altos a diferencia de los estudiantes con edades comprendidas entre los 20-24 años. Es destacable que en la subescala CAR (agente) los estudiantes de 30 años 0 más obtuvieron valores más altos que los de entre 20-24 años no siendo así con otros rangos de edad. Estos datos están en cierta concordancia con lo encontrado en estudios anteriores ya que al iniciar los estudios es cuando se evidencia mayor presencia de intimidación, lo que parece deberse a la dificultad para tener dinámicas de los grupos y la participación de los estudiantes en los mismos (Hoyos et al., 2009; Hoyos et al., 2012). La procedencia de los estudiantes indicó que no existían diferencias de medias en ninguna de las subescalas.

En lo referente al nivel de estudios que estaban cursando los estudiantes, cabe destacar que los estudiantes de Grado obtuvieron valores más altos que los estudiantes de Máster en las tres subescalas. Por otro lado, en la subescala CA (objeto) los estudiantes de otro tipo de formación obtuvieron valores más altos que los estudiantes de Máster. Al igual que ocurría con la edad, el nivel de estudios puede estar relacionado con el hecho de iniciar estudios de Grado u otro tipo de formación debido a la dificultad para tener dinámicas de los grupos (Hoyos et al., 2009; Hoyos et al., 2012). 
Atendiendo a la facultad a la que pertenecían los estudios en los cuales estaban matriculados los estudiantes, los resultados mostraron que los estudiantes de $\mathrm{Hu}$ manidades, Ciencias Sociales y Ciencias de la Salud obtuvieron valores más altos en comparación con los estudiantes de Ciencias en todas las subescalas. Del mismo modo, los estudiantes de Ciencias de la Salud obtuvieron valores más altos que los estudiantes de Ciencias Sociales en la subescala OBC. Los estudios anteriores no evidenciaron la existencia de diferencias en función de la facultad lo cual pueda deberse a que se limitaron a una 0 dos tipos de facultad (Molero et al., 2014; Paredes et al., 2010; Yubero et al., 2017).

A la hora de predecir CA, CAR y OBC se obtuvo que existía una relación entre el hecho de haber sido objeto de ciberacoso, agente de ciberacoso y observador de ciberacoso. Se ha de tener en cuenta la importancia de la pertenencia a una determinada facultad para CAR y CA para el nivel de estudios. Nuestro estudio ha permitido tener una perspectiva más amplia de la intimidación en el ámbito universitario ya que se ha centrado en analizar el tipo de facultad según se perteneciese a Humanidades, Ciencias Sociales y de la Educación, Ciencias de la Salud y Ciencias así como el tipo de estudios (Grado, Máster u otro tipo de formación).

\section{Conclusión}

Por lo tanto, conociendo el origen del acoso escolar es posible contextualizarlo en la comunidad universitaria y por ello promover actuaciones de prevención o de in- tervención, en su caso. Cabe destacar que no se trata de situaciones alarmantes pero sí preocupantes debido a las incidencias encontradas (Hoyos et al., 2009) y al impacto emocional (Walker, 2015). Se requiere implementar medidas preventivas y/o de intervención que permitan analizar cómo dicha problemática afecta al proceso académico y a las relaciones interpersonales en los diferentes campos educativos (García-Peña et al., 2013; Myers y Cowie, 2017). Para ello es necesario promover campañas de sensibilización, siendo necesario reforzar la educación emocional así como la adquisición de habilidades sociales (Sastre, 2016) y los valores de convivencia (Del Rey et al., 2012), tolerancia a las formas de agresión, difusión de las responsabilidad... (Hoyos et al., 2012); generar estrategias para evitar la agresión, fomentar la conducta prosocial, la empatía, el control emocional (Garaigordobil, 2015; Gómez-Ortiz, Romera-Félix y Ortega-Ruiz, 2017; Trujillo y et al., 2016). Del mismo modo, es necesario educar en todos los niveles escolares en el uso seguro de las TIC, haciendo especial hincapié en los principios morales y éticos en contra de la violencia (Ortega-Ruiz y Zych, 2016). Del Rey, Casas et al. (2012), aluden la importancia de que la institución educativa juega un papel importante en la materia de competencia tecnológica y ello supone que dicha competencia se debe articular con la competencia para la autonomía personal, aprender a aprender... Por ello, es fundamental promover la cibersocialización con programas como, por ejemplo, ConRed (Del Rey, Casas et al., 2012); o Ciberprogram 2.0 (Garaigordobil y Martínez-Valderrey, 2014). Siendo necesario promover la inclusión del alumnado, 
la convivencia y la ciberconvivencia (Garaigordobil, 2015; Llorent et al., 2016) incluso a través de la gamificación (Álvarez-Bermejo, Belmonte-Ureña, Martos-Martínez, Barragán-Martín y Simón-Márquez, 2016). Es necesario promover la supervisión parental del acceso a internet fomentando vínculos de apegos seguros (Bernal y Angulo, 2013; Garaigordobil, 2015). Por lo tanto, el contexto universitario debe asumir la importancia de incentivar la convivencia y el bienestar universitario (Cassidy et al., 2017) así como la información legal para los víctimas (Myers y Cowie, 2017). Asimismo, es relevante la implicación de toda la comunidad educativa.

Como prospectivas sería adecuado llevar a cabo estudios longitudinales así como sería conveniente incluir otras variables de interés como el rendimiento de los estudiantes universitarios y su implicación en conductas agresivas (Molero et al., 2014); analizar si se produce acoso hacia el profesorado, incluso el clima escolar y familiar (López, 2017) o del profesorado hacia el alumnado, investigar el acoso entre las relaciones de pareja (Durán y Martínez-Pecino, 2015) así como entre los estudiantes del colectivo de gais y lesbianas (LGTB) (Walker, 2015); analizar la aparición con otros comportamientos violentos asociados (Ortega-Barón et al., 2017); tener en cuenta el rol de víctima agresora ya que se ha evidenciado que en el contexto universitario también se da (Trujillo et al., 2016), el punto de vista del profesorado (Cassidy et al., 2017), o el rol de las prácticas docentes en la prevención de violencia entre iguales (Valdés-Cuervo, Martínez-Ferrer y Carlos-Martínez, 2018).
Entre las limitaciones de este estudio habría que tener en cuenta que se trata de un estudio transversal. Por otro lado, habría que tener en cuenta las limitaciones de haber utilizado como medidas el autoinforme ya que podría estar influido por la deseabilidad social. Finalmente, debido a que el porcentaje de varianza explicado no es muy alto, sería deseable utilizar a su vez otros instrumentos de evaluación que permitan saber qué otras variables están influyendo.

\section{Referencias bibliográficas}

Álvarez-Bermejo, J. A., Belmonte-Ureña, L. J., Martos-Martínez, A., Barragán-Martín, A. B. y Simón-Márquez, M. D. M (2016). System to Detect Racial-Based Bullying through Gamification. Frontiers Psychology, 7 (1791). doi: https://doi.org/10.3389/fpsyg.2016.01791

Álvarez-García, D., Barreiro-Collazo, A., Núñez, J. C. y Dobarro, A. (2016). Validity and reliability of the Cyber-aggression Questionnaire for Adolescents (CYBA). The European Journal of Psychology Applied to Legal Context, 8 (2), 69-77. doi: https://doi.org/10.1016/j.ejpal.2016.02.003

Álvarez-García, D., Núñez, J. C., Dobarro, A. y Rodríguez (2016). Risk factors associated with cybervictimization in adolescence. International Journal of Clinical and Health Psychology, 15, 226-235.

Anguiano-Carrasco, C. y Vigil-Colet A. (2011). Assessing indirect aggresion in aggressors and targets: Spanish adaptation of the Indirect Aggression Scales. Psicothema, 23 (1), 146-152.

Arnaiz, P., Cerezo, F., Giménez A. M. y Maquilón, J. J. (2016). Conductas de ciberadicción y experiencias de cyberbullying entre adolescentes. Anales de Psicología, 32 (3), 761-769. doi: https://doi.org/10.6018/analesps.32.3.217461

Bernal, C. y Angulo, F. (2013). Interacciones de los jóvenes andaluces en las redes sociales. $\mathrm{Co}$ municar. Revista Científica de Comunicación y Educación, 20 (40), 25-30. doi: https://doi. org/10.3916/C40-2013-02-02 
Buelga, S., Cava, M. J. y Musitu, G. (2010). Cyberbullying: victimización entre los adolescentes a través del teléfono móvil y de Internet. Psicothema, 22 (4), 784-789.

Cassidy, W., Faucher, C. y Jackson, M. (2017). Adversity in University: Cyberbullying and Its Impacts on Students, Faculty and Administrators. International Journal of Environmental Research and Public Health, 14 (8), 888. doi: https://doi.org/10.3390/ijerph14080888

Cerezo, F., Martín, C., Martínez, J. P., Méndez, I. y Ruiz, C. (2016). Diseño y puesta en marcha del Cuestionario sobre Acoso entre Estudiantes Universitarios (QAUE). Comunicación presentada en el VII Congreso Internacional de Psicología y Educación, Asociación Científica de Psicología y Educación, Alicante, España.

Cohen, J. (1988). Statistical power analysis for the behavioral sciences Statistical Power Analysis for the Behavioral Sciences. Hillsdale, NJ: Lawrence Erlbaum.

Crosslin, K. y Golman, K. (2014). "Maybe you don't want to face it". College students' perspectives on ciberbullying. Computers in Human Behavior, 41, 14-20. doi: https://doi.org/10.1016/j. chb.2014.09.007

Del Rey, R., Casas, J. A. y Ortega-Ruiz, R. (2012). El programa ConRed, una práctica basada en la evidencia. Comunicar. Revista científica de Educación y Comunicación, 20 (39), 129-138.

Del Rey, R., Elipe, P. y Ortega-Ruiz, R. (2012). Bullying and ciberbullying: overlapping and predictive value of the co-occurrence. Psicothema, 24 (4), 608-613.

Durán, M. y Martínez-Pecino, R. (2015). Ciberacoso mediante teléfono móvil e Internet en las relaciones de noviazgo entrejóvenes. Comunicar. Revista cientifica de Educación y Comunicación, 22 (44), 159-167. doi: http://dx.doi.org/10.3916/C44-2015-17

Faucher, C., Jackson, M. y Cassidy, W. (2014). Cyberbullying among University Students: Gendered Experiences, Impacts, and Perspectives. Education Research International, 2014 (698545). doi: http://dx.doi.org/10.1155/2014/698545

Garaigordobil, M. (2011). Prevalencia y consecuencias del cyberbullying: una revisión. International Journal of Psychology and Psychological Therapy, 11 (2), 233-254.
Garaigordobil, M. (2015). Ciberbullying en adolescentes y jóvenes del País Vasco: Cambios con la edad. Anales de Psicología, 31 (3), 1069-1076. doi: https://doi.org/10.6018/analesps.31.3.179151

Garaigordobil, M. y Martínez-Valderrey, V. M. (2014). Efecto del Cyberprogram 2.0 sobre la reducción de la victimización y la mejora de la competencia social en la adolescencia. Revista de Psicodidáctica, 19 (2), 289-305. doi: https:// doi.org/10.1387/RevPsicodidact.10239

García-Peña, J. J., Moncada Ortiz, R. M. y Quintero Gil, J. (2013). El bullying y el suicidio en el escenario universitario. Revista Colombiana de Ciencias Sociales, 4 (2), 298-310.

Gómez-Ortiz, 0., Romera-Félix, E. M. y Ortega-Ruiz, R. (2017). Multidimensionalidad de la competencia social: medición del constructo y su relación con los roles del bullying. Revista de Psicodidáctica, 22 (1), 37-44. doi: https://doi. org/10.1016/S1136-1034(17)30042-4

Hernández Prados, M. A. y Solano Fernández, M. I. (2007). Ciberbullying, un problema de acoso escolar. RIED: Revista Iberoamericana de Estudios a Distancia, 10 (1), 17-36.

Hoyos, O. L. R., Llanos M. M. y Valega, S. J. (2012). El maltrato entre iguales por abuso de poder en el contexto universitario: incidencia, manifestaciones y estrategias de solución. Universitas Psychologica, 11 (3), 793-802.

Hoyos, O. L. R., Romero, L., Valega, S. J. y Molinares, C. (2009). El maltrato entre iguales por abuso de poder y exclusión social en estudiantes de una universidad privada de la ciudad de Barranquilla. Pensamiento Psicológico, 6 (13), 109-125.

Kokkinos, C. M., Antoniadou, N. y Markos, A. (2014). Cyber-bullying: An investigation of the psychological profile of university student participants. Journal of Applied Developmental Pscychology, 35, 204-2014. doi: https://doi. org/10.1016/j.appdev.2014.04.001

Llorent, V. J., Ortega-Ruiz, R. y Zych, I. (2016). Bullying and Cyberbullying in Minorities: Are They More Vulnerable than the Majority Group? Frontiers in Psychology, 7 (1507). doi: https://doi.org/10.3389/fpsyg.2016.01507

López, M. G. (2017). Influencia del clima escolar y familiar en el acoso escolar y cibernético de universitarios. Revista Mexicana de Investigación en Psicología, 9 (1), 31-44. 
Martínez, J. P., Méndez, I., Ruiz-Esteban, C. y Cerezo, F. (2018). (Manuscrito inédito). Validación y fiabilidad del cuestionario sobre Acoso entre Estudiantes Universitarios (QAEU). Departamento de Psicología Evolutiva y de la Educación, Universidad de Murcia, España.

McDougall, L. (1999). A Study of Bullying in Further Education. Pastoral Care in Education, 17 (2), 31-37.

Molero, M. M., Gázquez, J. J., Pérez-Fuentes, M. C. y Soler, F. J. (2014). Rendimiento académico y conducta agresiva en estudiantes universitarios. European Journal of Child development, Education and Psychopathology, 2 (2), 69-79.

Myers, C-A. y Cowie, H. (2017). Bullying at University: The Social and Legal Contexts of Cyberbullying Among University Students. Journal of Cross-Cultural Psychology, 48 (8), 1172-1182. doi: https://doi.org/10.1177/0022022116684208

Olweus, D. (2013). Schoolbullying: development and some important challenges. Annual Review of Clinical Psychology, 9, 751-780. doi: https://doi. org/10.1146/annurev-clinpsy-050212-185516

Ortega-Barón, J., Buelga, S., Cava, M. J. y Torralba, E. (2017). Violencia escolar y actitud hacia la autoridad de estudiantes agresores de ciberbullying. Revista de Psicodidáctica, 22 (1), 2328. doi: 10.1387/RevPsicodidact.16398

Ortega-Ruiz, R. (2015). Convivencia y Ciberconvivencia. Un Modelo Educativo para la Prevención del Acoso y el Ciberacoso Escolar. Madrid: Machado Libros.

Ortega-Ruiz, R. y Zych, I. (2016). La ciberconducta y la psicología educativa: retos y riesgos. Psicología Educativa 22 (1), 1-4. doi: https://doi. org/10.1016/j.pse.2016.04.001

Paredes, 0., Sanabria-Ferrand, P. A., González-Quevedo, L. A. y Moreno Rehalpe, S. P. (2010). "Bullying" en las facultades de Medicina colombianas, mito o realidad. Revista Med, 18 (2), 161-172. doi: https://doi.org/10.18359/ rmed.1309

Sastre, A. (Coord.) (2016). Yo a eso no juego. Bullying y ciberbullying en la infancia. Madrid: Save the Children España Recuperado de http://www.observatoriodelainfancia.es/oia/ esp/descargar.aspx?id=4883\&tipo=documento (Consultado el 03/04/2019).
Torres Mora, M. T. (2010). El problema de la violencia entre universitarios abordado desde el enfoque de la Investigación-Participación-Acción. Investigación Universitaria Multidisciplinaria, 9 (9), 27-36.

Trujillo, J. J. y Romero-Acosta, K. (2016) Variables que evidencian el bullying en un contexto universitario. Revista Encuentros, Universidad Autónoma del Caribe, 14 (1), 41-54. doi: http:// dx.doi.org/10.15665/re.v14i1.668

Valdés-Cuervo, A. A., Martínez-Ferrer, B. y Carlos-Martínez, E. A. (2018). El rol de las prácticas docentes en la prevención de la violencia escolar entre pares. Revista de Psicodidáctica, 23 (1), 33-38. doi: https://doi.org/10.1016/j. psicod.2017.05.006

Walker, C. (2015). An Analysis of Cyberbullying Among Sexual Minority University Students. Journal of Higher Education Theory and Practice, 15 (7), 44-50.

Yubero, S., Navarro, R., Elche, M., Larrañaga, E. y Ovejero, A. (2017). Cyberbullying victimization in higher education: An exploratory analysis of its association with social and emotional factors among Spanish students. Computers in Human Behavior. doi: https://doi.org/10.1016/j. chb.2017.05.037

Zych, I., Ortega-Ruiz, R. y Marín-López, I. (2016). Cyberbullying: a systematic review of research, its prevalence and assessment issues in Spanish studies. Psicología Educativa, 22 (1), 5-18. doi: https://doi.org/10.1016/j.pse.2016.03.002

\section{Biografía de los autores}

Inmaculada Méndez Mateo es Licenciada y Doctora en Psicología por la Universidad de Murcia. Es Profesora Ayudante Doctor en esta universidad y Coordinadora de Calidad en ISEN Centro Universitario. Entre sus líneas de investigación destacan: conductas de riesgo social y para la salud en la infancia, adolescencia y jóvenes (consumo de drogas, acoso, etc.) así como el envejecimiento activo y saludable.

(iD https://orcid.org/0000-0001-8458-5314 
Cecilia Ruiz Esteban es Licenciada en Ciencias de la Educación por la Universidad Complutense de Madrid, Master of Science in Education por la Bucknell University y Doctora en Psicología por la Universidad de Murcia. Es Profesora Titular en esta universidad e Investigadora Principal del Grupo de investigación EIPSED (Equipo de Investigación en Psicología de la Educación) de la Universidad de Murcia. Sus principales líneas de investigación versan sobre acoso y ciberacoso entre iguales, variables personales del proceso de enseñanza-aprendizaje y la calidad de la educación superior.

(iD https://orcid.org/0000-0002-5836-331X

Juan Pedro Martínez Ramón es Licenciado y Doctor en Psicología por la Universidad de Murcia. Licenciado en Psico-

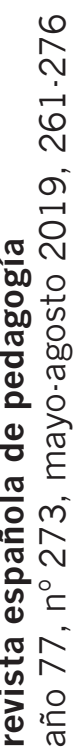
pedagogía por la Universidad Nacional de
Educación a Distancia (UNED). Profesor asociado en el Departamento de Psicología Evolutiva y de la Educación de la Universidad de Murcia. Sus líneas de investigación giran en torno a la convivencia escolar, estrés del profesorado y atención a la diversidad.

(iD https://orcid.org/0000-0003-1770-6598

Fuensanta Cerezo Ramírez es Profesora Colaboradora Honoraria de la Universidad de Murcia. Sus investigaciones se centran en el bullying, la formación del profesorado, la victimización escolar, la percepción de profesores, alumnos y padres sobre los planes de convivencia escolar el desarrollo de un programa telemático para mejorar la convivencia a través de la inteligencia emocional, bullying y ciberbullying en las aulas universitarias.

(iD https://orcid.org/0000-0002-3160-9930 


\section{revista española de pedagogía \\ año 77, n 273, mayo-agosto 2019 \\ Spanish Journal of Pedagogy \\ year 77, n. 273, May-August 2019 \\ Sumario* \\ Table of Contents **}

\section{Respuestas educativas \\ y cívico-penales a los comportamientos antisociales}

\section{Educational and civic-penal responses to antisocial behavior}

Editor invitado: Fernando Gil Cantero Guest editor: Fernando Gil Cantero

\section{Fernando Gil Cantero}

Presentación: Respuestas educativas y cívico.penales a los comportamientos antisociales

Introduction: Educational and civic-penal responses to antisocial behavior

David Reyero, Fernando Gil Cantero

La educación que limita es la que libera

Education that limits is education that frees

Maria José Bernuz Beneitez, Esther Fernández Molina La pedagogía de la justicia de menores: sobre una justicia adaptada a los menores

The pedagogy of juvenile justice: a child-friendly justice

Marina Martins, Carolina Carvalho

¿En qué mienten los adolescentes?

What do teenagers lie about?
Inmaculada Méndez, Cecilia Ruiz Esteban, Juan Pedro Martínez, Fuensanta Cerezo

Ciberacoso según características sociodemográficas y académicas en estudiantes universitarios

Cyberbullying according to sociodemographic and academic characteristics among university students

261

Irene Montiel, José R. Agustina

Retos educativos ante los riesgos emergentes en el ciberespacio: claves para una adecuada prevención de la cibervictimización en menores

Educational challenges of emerging risks in cyberspace: foundations of an appropriate strategy for preventing online child victimization

Maialen Garmendia Larrañaga, Estefanía Jiménez Iglesias, Nekane Larrañaga Aizpuru Bullying y ciberbullying: victimización, acoso y daño.

Necesidad de intervenir en el entorno escolar Bullying and cyberbullying: victimisation, harassment, and harm. The need to intervene in the educational centre

Ana Rosser-Limiñana, Raquel Surià-Martínez Adaptación escolar y problemas comportamentales y emocionales en menores expuestos a violencia de género

School adaptation and behavioural and emotional problems in minors exposed to gender violence

\footnotetext{
* Todos los artículos están también publicados en inglés en la página web de la revista: https://revistadepedagogia.org.

** All the articles are also published in English on the web page of the journal: https://revistadepedagogia.org.
} 
Fanny T. Añaños-Bedriñana, Miguel Melendro, Rocío

Raya Miranda

Mujeres jóvenes con medidas de protección y judiciales y sus tránsitos hacia la prisión

Young women with protective and judicial measures and their transit towards prison

333

\section{Reseñas bibliográficas}

\section{Esteban Bara, F. Ética del profesorado (Juan} García Gutiérrez). García Amilburu, M., Bernal, A. y González Martín, M. R. Antropología de la educación. La especie educable (Yaiza Sánchez Pérez). Rose, D. y Martin, J. R. Leer para aprender. Lectura y escritura en las áreas del currículo (Francisco Lorenzo Bergillos). Buxarrais, M. R. y Vilafranca, I. (Coords.). Una mirada femenina de la educación moral (Eric Ortega González).

351

\section{Informaciones}

«ECER conference Education in an Era of Risk.the Role of Educational Research for the Futurey - de la European Educational Research Association (EERA)-; III Congreso «European Liberal Arts and Core Texts Educations; VIII Congreso del Jubilee Centre for Character and Virtues: «Virtues and the Flourishing Lifen; XLV Congreso Internacional de AME 2019: «Morality and Ethics for the Digital World»; III Premio Ricardo Marín Ibáñez; Una visita a la hemeroteca (profesores youtubers) (Daniel Pattier); Una visita a la red (David Reyero).

\section{Instrucciones para los autores}

Instructions for authors

375

ISSN: 0034-9461 (Impreso), 2174-0909 (Online)

https://revistadepedagogia.org/

Depósito legal: M. 6.020 - 1958

INDUSTRIA GRÁFICA ANZOS, S.L. Fuenlabrada - Madrid 


\title{
Cyberbullying according to sociodemographic and academic characteristics among university students \\ Ciberacoso según características sociodemográficas y académicas en estudiantes universitarios
}

\author{
Inmaculada MÉNDEZ, PhD. Assistant Professor. Universidad de Murcia (inmamendez@um.es). \\ Cecilia RUIZ ESTEBAN, PhD. Lecturer. Universidad de Murcia (cruiz@um.es). \\ Juan Pedro MARTÍNEZ, PhD. Associate Lecturer. Universidad de Murcia (juanpedromartinezramon@um.es). \\ Fuensanta CEREZO, PhD. Assistant Honorary Lecturer. Universidad de Murcia (fcerezo@um.es).
}

\section{Abstract:}

There is evidence of the existence of both bullying and cyberbullying in the university environment. The aim of this study is to analyse the differences between cyberbullying roles (target, perpetrator, and bystander) according to sociodemographic and academic variables (sex, age, origin, level of studies, and faculty). The participants were 765 young students ( $72.9 \%$ women) from a university of the southeast of Spain $(83.7 \%$ undergraduate degree, $15.2 \%$ Master's degree, and $1.1 \%$ other studies) from different faculties. The Questionnaire on Harassment among University Students tool was used. The results of the study determined that women, people aged under 20 , undergraduate degree students, and Humanities, Social Sciences, and Health Sciences stu- dents obtained higher values in the sub-scales evaluated. Among the roles of those involved, bystanders stood out, followed by perpetrators and victims. Regression analysis showed a relationship between being the target, perpetrator, and/or bystander of cyberbullying. The study will make it possible to focus on those sociodemographic variables that turned out to be significant as well as the relationship between the cyberbullying roles in the face of prevention and intervention programs for each of the roles. The university context must assume the importance of promoting coexistence and university welfare. The involvement of the entire educational community is also relevant.

Keywords: cybernetics, universities, university faculty, education, cyberbullying.

Revision accepted: 2019-02-11.

This is the English version of an article originally printed in Spanish in issue 273 of the revista española de pedagogía. For this reason, the abbreviation EV has been added to the page numbers. Please, cite this article as follows: Méndez, I., Ruiz Esteban, C., Martínez, J. P., \& Cerezo, F. (2019). Ciberacoso según características sociodemográficas y académicas en estudiantes universitarios | Cyberbullying according to sociodemographic and academic characteristics among university students. Revista Española de Pedagogía, 77 (273), 261-276. doi: https:// doi.org/10.22550/REP77-2-2019-06 


\section{Resumen:}

Existe evidencia de la aparición del acoso escolar en el ámbito universitario tanto de manera presencial como a través de medios tecnológicos. El objetivo del estudio fue analizar las diferencias entre los roles del ciberacoso (objeto, agente y observador) en función de variables sociodemográficas y académicas (sexo, edad, procedencia, nivel de estudios y Facultad). Los participantes del estudio fueron 765 jóvenes estudiantes $(72.9 \%$ mujeres) de una universidad del sureste español (83.7\% estudios de Grado, el 15.2\% de Máster y el $1.1 \%$ otros estudios) pertenecientes a diferentes Facultades (Humanidades, Ciencias Sociales y de la Educación, Ciencias de la Salud y Ciencias). El instrumento utilizado fue el Cuestionario sobre Acoso entre Estudiantes Universitarios. Los resultados del estudio determinaron que las mujeres, los menores de 20 años, los estudiantes de Grado, los estu- diantes de Humanidades, Ciencias Sociales y Ciencias de la Salud obtuvieron valores más altos en las subescalas evaluadas. Entre los roles de los implicados destacaban los observadores seguido de agresores y de víctimas. $\mathrm{El}$ análisis de regresión determinó la relación entre el hecho de haber sido objeto de ciberacoso, agente de ciberacoso y observador de ciberacoso. El estudio permitirá centrarse en aquellas variables sociodemográficas que resultaron ser significativas, así como la relación entre los roles del ciberacoso de cara a programas de prevención e intervención en cada uno de sus roles. El contexto universitario debe asumir la importancia de promover la convivencia y el bienestar universitario. Asimismo, es relevante la implicación de toda la comunidad educativa.

Descriptores: cibernética, universidad, facultad universitaria, educación, ciberacoso.

\section{Introduction}

Problems of intimidation or harassment among peers can appear throughout the educational period (Ortega-Ruiz, 2015), mainly in cultural/ethnic, sexual minorities and minorities of other types (Llorent, Ortega-Ruiz, \& Zych, 2016; Walker, 2015). Such situations are categorised by being intentional in nature and by being situations repeated over time carried out by an individual or a group against victims unable to defend themselves, with a resulting imbalance of power (Olweus, 2013).
Cyberbullying involves using ICT -Information and Communication Technologies - to harass other peers, mainly by using the Internet and mobile phones. The methods generally used are text messages, phone calls, recording and publication of attacks and humiliations, social networks, and so on (Garaigordobil, 2015), repeated over time, intentionally and with an imbalance of power (Zych, Ortega-Ruiz, \& Marín-López, 2016). In general, there are three groups of roles directly involved in this issue: victim or target, perpetrator, and bystander, either directly or through cyberbullying. 
Cyberbullying is often covered up (Hernández \& Solano, 2007), and the concealment of the aggressor's identity facilitates impunity and increases the victim's defencelessness. Electronic attacks are spread rapidly to a great number of people who can share them in turn (Buelga, Cava, $\&$ Musitu, 2010).

The virtual world offers possibilities as well as risks, such as, for instance, Internet addiction or cyberbullying (Arnaiz, Cerezo, Giménez, \& Maquilón, 2016; Del Rey, Casas, \& Ortega-Ruiz, 2012; Myers $\&$ Cowie, 2017). Del Rey et al. (2012) state that overuse of new technologies is a risk that may affect students' quality of life since it generates a situation of dependence. Several studies have demonstrated that cyberbullying may have legal and highly negative psychological consequences for the students involved (Álvarez-García, Barreiro-Collazo, Núñez, \& Dobarro, 2016). Ortega-Barón, Buelga, Cava, and Torralba (2017) showed that students who had been cyberbullies, either continuously or occasionally, had previously broken the rules, that is to say, they had been involved in other proscribed behaviour.

Among protective factors, it is important to highlight self-esteem as protection against occasional cyber-victimisation arising from owning a mobile phone, playing online games, and frequency of Internet use on week days. Cyber-victimisation, either continuous or occasional, is mainly found among girls and with an average age of 14 (Álvarez-García, Núñez, Dobarro, \& Rodríguez, 2016). Similarly, Arnaiz et al.
(2016) affirm that family supervision is a protective factor.

The coexistence of traditional bullying and cyberbullying suggests that involvement in cyberbullying may be predicted by involvement in traditional bullying (cyber-victimisation and cyber-aggression) (Del Rey, Elipe, \& Ortega-Ruiz, 2012; Yubero, Navarro, Elche, Larrañaga, \& Ovejero, 2017). Save the Children's report (Sastre, 2016) on bullying and cyberbullying showed that $9.3 \%$ of students in Spain had been victims of school bullying and $6.9 \%$ victims of cyberbullying. Among the ways it manifests itself, insults were the most common form (six out of ten students had used insults and more than two out of ten had been insulted frequently), followed by other forms such as rumours, theft, threats, physical mistreatment, or exclusion. Moreover, 5.4\% had suffered cyber-bullying and $3.3 \%$ had committed cyberbullying. This shows that the prevalence of cyberbullying is increasing (Álvarez-García et al., 2016; Garaigordobil, 2011, 2015).

Therefore, cyberbullying is a social problem which goes beyond the borders of the educational contexts of primary and secondary education, affecting other types of population, such as university circles, with the same devastating effects (Crosslin \& Golman, 2014; García-Peña, Moncada, \& Quintero, 2013; Torres-Mora, 2010; Walker, 2015).

Until recently, the risk in the university population had not been considered, as university students were ascribed a cer- 
tain psychosocial maturity which would minimise relationship problems among them. It must be noted that intimidation among young university students is not only a social issue but also a problem of public health.

It follows from the above that bullying is not exclusively a phenomenon of the of the primary and secondary education context since it presents itself as a relationship problem among peers which can arise in other academic locations such as universities, and such abuse of power even appears through new technologies (cyberbullying). It is a serious problem which has negative consequences for the development of university students (Cassidy, Faucher, \& Jackson, 2017; Crosslin \& Golman, 2014; García-Peña et al., 2013; Walker, 2015).

In the university context, there is a series of factors that cause stress, anxiety, depressive symptoms, and other problems. One of these factors is intimidation or harassment among students (García-Peña et al., 2013). The factors which contribute to the appearance of intimidation in the university context include different academic abilities, peer-groups, and students with special needs (McDougal, 1999).

The existence of lower levels of physical aggression among university students in comparison to other educational levels has been shown, while rates of other types of hostility such as verbal abuse (taunts, insults, etc.), social exclusion through alienation, denigration, abuse in group work, categorization by physical features and economic status, among others, are greater (Hoyos, Romero, Valega, \& Molinares, 2009; López, 2017; Paredes, Sanabria-Ferrand, González-Quevedo, \& Moreno Rehalpe, 2010; Torres-Mora, 2010; Trujillo \& Romero-Acosta, 2016). Among the roles of those involved, bystanders are most apparent, followed by bullies and victims (Hoyos et al., 2009; Paredes et al., 2010; Trujillo et al., 2016). Both men and women are actors in the different types of abuse (Anguiano-Carrasco \& Vigil-Colet, 2011; Faucher, Jackson, \& Cassidy, 2014; Hoyos et al., 2009; Kokkinos, Antoniadou, \& Markos, 2014). Specifically, men are usually more involved as victim than women, although not in all of its manifestations, while women appear as bystanders. In relation to bullies, women appeared as bullies in different ways (ignoring, disparaging, etc.) than men (name-calling, insulting, etc.) (Hoyos et al., 2009; Hoyos et al., 2012). With reference to the courses, Molero, Gázquez, Pérez-Fuentes and Soler (2014) did not find differences between the types of degrees analysed (Primary Education, Early Childhood Education, and Psychology), nor did Paredes et al. (2010) who analysed situations of bullying in faculties of medicine in Colombia. In relation to age, there is a greater presence of abuse in more forms between 17 and 20 (Hoyos et al., 2009; Hoyos et al., 2012) which seems to derive from the difficulty of establishing group dynamics and each student's participation in them.

For this reason, the aim of this study is to analyse differences because of socio-demographic and academic variables (sex, 
age, origin, level of studies and faculty) among cyberbullying roles (victim of cyberbullying, perpetrator of cyberbullying, and bystander of the cyberaggression).

\section{Method}

\subsection{Participants}

The participants in the study were 765 young university students in southern Spain with ages ranging from under 20 to over $30,72.9 \%$ of whom were women (see Table 1), $93.5 \%$ of students were of Spanish origin. In relation to the type of studies, $83.7 \%$ were studying for an undergraduate degree, $15.2 \%$ for a Master's, and the others, other studies. The distribution by faculty was: $14 \%$ Humanities (Fine Art, Geography, History, Languages, Classical Philology, and others), 45.6\% Social Sciences and Education (Law, Economics, Social Work, Education, and others), 30.2\% Health Sciences (Medicine, Nursing, Psychology, Optometry, Physiotherapy, and others), and $10.2 \%$ Science (Physics, Chemistry, Biology, Computing, Engineering, and others).

TABLE 1. Distribution of participants.

\begin{tabular}{|l|c|c|}
\hline \multicolumn{1}{|c|}{ Age } & Men & Women \\
\hline Under 20 & $41(19.8 \%)$ & $182(32.8 \%)$ \\
\hline $20-24$ & $125(60.4 \%)$ & $276(49.7 \%)$ \\
\hline $25-29$ & $28(13.5 \%)$ & $58(10.5 \%)$ \\
\hline 30 or more & $13(6.3 \%)$ & $39(7 \%)$ \\
\hline
\end{tabular}

Source: Own elaboration.

\subsection{Instruments}

The instrument used was the Questionnaire on Harassment among University Students by Cerezo, Martín, Martínez, Méndez, and Ruiz (2016) validated by Martínez, Méndez, Ruiz and Cerezo (not published).

The instrument consists of 135 items. The first part measures socio-demographic and academic variables such as: age (under 20, between 20-24, between 25-29, 30 or more), gender (male/female), faculty where studies were pursued (Humanities, Social Sciences and Education, Health Sciences, and Sciences), type of studies (undergraduate degree, Master's, or others), year (1st, 2nd, 3rd, or 4th) and country of origin (Spain or other). The instrument then uses three scales:

a) As target of aggression.

b) As perpetrator of aggression.

c) As bystander of aggression. 
Each of these measures direct aggression (bullying) as well as cyber aggression (cyberbullying):

Scale a) As target of aggression: consists of 49 items distributed between bullying (type, who, place, time, reason) and cyberbullying (type, way or means, moment, reason, informer and support), it is made up of the sub-scales direct aggression (DA) (e.g., "I have suffered physical aggression") and target of cyberbullying (TCB) (e.g., "on occasion I have received cyberbullying through social networks").

Scale b) As perpetrator of aggression: has 35 items distributed between direct aggression or harassment (type, object or direction, moment, and cause) and cyberbullying (act, path, object, and motives), and it comprises the sub-scales perpetrator of direct aggressions (PDA) (e.g., "on occasion I have verbally harassed a partner with insults, threat, etc.") and perpetrator of cyberbullying (PCB) (e.g., "on occasion I have done cyberbullying through offensive calls").

Scale c) As bystander of the aggression: consists of 45 items distributed between observation of direct aggression or harassment (type, object, moment and motives) and cyberbullying (type, route, excluded, causes, informed and help) made up of the sub-scales of bystander of direct aggressions (BDA) (e.g., "on occasion, I have seen another student assaulted, the aggressions were physical") and bystander of cyberbullying (BCB) (e.g., "on occasion, I have observed cyberbullying -aggression to others through mobile phones or the Internet- through calls"). These sub-scales have appropriate Cronbach's Alpha values: as target of aggression .95; as perpetrators of aggression .94 and as bystander of the aggression .96. In this study, the sub-scales relating to cyberbullying (TCB, PCB and $\mathrm{BCB}$ ) were selected.

\subsection{Process}

Participants were selected taking into account the faculty in which they were enrolled in the 2015-2016 academic year in such a way that the representativeness of each group of the 20 faculties at the University in southern Spain was guaranteed (Humanities, Social Sciences and Education, Health Sciences, and Sciences) with a confidence level of $95 \%$. It was necessary to ask for permission and collaboration of the teachers in charge of the undergraduate degrees, Master's, or other studies selected so the questionnaires could be administered in person or online through the course's virtual classroom $(28.48 \%)$. Administering the questionnaire took between 15 and 20 minutes. Data confidentiality and anonymity were guaranteed during the completion of the instrument. The protocol was approved by the Ethics Committee for Clinical Research of the Universidad de Murcia. The 
study was performed in accordance with the approved guidelines and the Declaration of Helsinki.

\subsection{Data Analysis}

To analyse cyberbullying roles according to socio-demographic characteristics, a mean difference was calculated (Student's $t$ ) for our independent samples to determine if there were differences relating to gender and origin for each of the sub-scales and Cohen's d (1988) was calculated for the effect size. To analyse the mean differences by level of studies, the faculty in which they were enrolled, and age (in ranges), an analysis of variance (ANOVA) was used for each of the subscales. Comparisons with the Bonferroni Post-Hoc Test were used. In addition, the Brown-Forsythe test was used when Levene's test could not assess the equality of variances. Hierarchical regression analyses were used to contrast the predictive power of the groups of independent variables (socio-demographic variables such as age, gender, faculty, level of studies, origin) in relation to the dependent variable under study (TCB, PCB, BCB) as well as the relations between them and the "enter" method. Analyses were carried out using the SPSS v.21 program.

\subsection{Results}

$14.4 \%$ of the students had suffered cyberbullying, $7.3 \%$ had committed cyberbullying, and $17.4 \%$ had witnessed cyberbullying.

Table 2 shows the differences by gender in the QAEU sub-scales. Student's t-test showed significant mean differences in the TCB, $\mathrm{PCB}$, and $\mathrm{BCB}$ sub-scales with higher values in women and a low effect size $(d=-.20)$.

Secondly, Table 3 shows the differences by age range in the QAEU sub-scales in the one-way ANOVA test. The post hoc tests revealed that there were mean differences between students younger than 20 and students aged between 20 and 24 in the three sub-scales. In the PCB sub-scale, there were mean differences between students aged between 20 and 24 and students aged 30 or more, being higher in the latter.

Table 4 shows the differences by place of origin in the QAEU sub-scales. Student's t-test did not show significant mean differences in the TCB, PCB, and BCB sub-scales.

Table 5 shows the study results by level of studies. The post hoc tests showed that there were significant differences between undergraduate degree and Master's students in the three sub-scales (TCB, PCB, and $\mathrm{BCB}$ ), the figures being lower for the latter. Likewise, in the TCB sub-scale, there were mean differences between Master's students and those enrolled in other types of training, the figures being higher for the latter.

Table 6 shows the results relating to the faculty of the course on which the students were enrolled. The post hoc tests showed that there were significant mean differences with Science students (who had lower values) and students from Humanities, Social Sciences, and Health Sciences in the 
TCB, PCB, and BCB sub-scales. Moreover, in the $\mathrm{OBC}$ sub-scale, there were mean differences between Social Sciences and Health Sciences students, with the former being higher.

Finally, the regression analysis, using TCB as criterion and as predictor variables those relating to the sociodemographic and academic variables (age, gender, faculty, level of studies, and origin) as well as PCB and BCB explained $65.8 \%$ of the variance. The standardised Beta regression coefficient showed that, among all the predictor variables, level of studies (Beta $=-.062 ; \mathrm{t}=-2.606$; $\mathrm{p}=.009), \mathrm{PCB}($ Beta $=.474 ; \mathrm{t}=14.458$; $\mathrm{p}=.000$ ), and $\mathrm{BCB}$ were significant. Similarly, the regression analysis which used PCB as its criterion and as predictor variables those relating to sociode- mographic and academic variables (age, gender, faculty, level of studies and origin) as well as TCB and BCB, explained $66.6 \%$ of the variance. The standardised Beta regression coefficient showed that, among all the predictor variables, faculty (Beta $=-.048 ; \mathrm{t}=-2.163 ; \mathrm{p}=.031), \mathrm{TCB}$ $($ Beta $=.459 ; \mathrm{t}=14.458 ; \mathrm{p}=.000)$, and BCB $($ Beta $=.409 ; \mathrm{t}=12.974 ; \mathrm{p}=.000)$ were significant. Also, the regression analysis with $\mathrm{BCB}$ as criterion and as predictor variables those relating to sociodemographic and academic variables (age, gender, faculty, level of studies, and origin) as well as TCB and PCB explained $63.6 \%$ of the variance. The standardised Beta regression coefficient showed that, among all the predictor variables, TCB $($ Beta $=.401 ; \mathrm{t}=11.615 ; \mathrm{p}=.000)$ and PCB $($ Beta $=.447 ; \mathrm{t}=12.974 ; \mathrm{p}=.000)$ were significant.

TABLE 2. Differences by gender in the QAEU sub-scales.

\begin{tabular}{|c|c|c|c|c|}
\hline \multirow{3}{*}{ QAEU SUB-SCALES } & \multicolumn{2}{|c|}{ GENDER } & & \\
\hline & MEN & WOMEN & & \\
\hline & $M(S D)$ & $M(S D)$ & $t$ & $p$ \\
\hline TCB & $12.24(14.81)$ & $15.28(15.62)$ & -2.243 & $.016^{*}$ \\
\hline PCB & $5.9(8.22)$ & $7.6(9.2)$ & -2.349 & $.019 *$ \\
\hline BCB & $11.91(16.08)$ & $15.26(17.43)$ & -2.501 & $.013 *$ \\
\hline
\end{tabular}

Note: $\mathrm{TCB}=$ target of cyberbullying, $\mathrm{PCB}=$ perpetrator of cyberbullying, $\mathrm{BCB}=$ bystander of cyberbullying.

Source: Own elaboration. 
TABLE 3. Differences by age range in the QAEU sub-scales.

\begin{tabular}{|c|c|c|c|}
\hline \multirow{2}{*}{ QAEU SUB-SCALES } & AGE & \multirow[b]{2}{*}{ Mean differences } & \multirow[b]{2}{*}{$p$} \\
\hline & $F$ & & \\
\hline TCB & $\begin{array}{l}\text { Brown-Forsythe } \\
F(3 ; 30.423)=3.633 \\
p=.013\end{array}$ & $\begin{array}{l}\text { Under } 20-20-24 \text { years }=3.60 \\
\text { Under } 20-25-29 \text { years }=3.40 \\
\text { Under } 20-30 \text { or more }=-1.35 \\
20-24 \text { years }-25-29 \text { years }=-.20 \\
20-24 \text { years- } 30 \text { or more }=-4.95 \\
25-29 \text { years }-30 \text { or more }=-4.75\end{array}$ & $\begin{array}{l}.031^{*} \\
\text { n.s. } \\
\text { n.s. } \\
\text { n.s. } \\
\text { n.s. } \\
\text { n.s. }\end{array}$ \\
\hline PCB & $\begin{array}{l}\text { Brown-Forsythe } \\
F(3 ; 280.899)=6.856 \\
p=.000\end{array}$ & $\begin{array}{l}\text { Under } 20-20-24 \text { years }=3.06 \\
\text { Under } 20-25-29 \text { years }=2.12 \\
\text { Under } 20-30 \text { or more }=-.77 \\
20-24 \text { years-25-29 years }=-.94 \\
20-24 \text { years-30 or more }=-3.83 \\
25-29 \text { years-30 or more }=-2.89\end{array}$ & $\begin{array}{l}.000 \\
\text { n.s. } \\
\text { n.s. } \\
\text { n.s. } \\
.019^{*} \\
\text { n.s. }\end{array}$ \\
\hline BCB & $\begin{array}{l}\text { Brown-Forsythe } \\
F(3 ; 322.231)=5.148 \\
p=.002\end{array}$ & $\begin{array}{l}\text { Under } 20 \text { years- } 20-24 \text { years }=5.52 \\
\text { Under } 20 \text { years- } 25-29 \text { years }=3.44 \\
\text { Under } 20 \text { years-30 or more }=1.49 \\
20-24 \text { years- } 25-29 \text { years }=-2.08 \\
20-24 \text { years-30 or more }=-4.04 \\
25-29 \text { years-30 or more }=-1.95\end{array}$ & $\begin{array}{l}.001^{*} \\
\text { n.s. } \\
\text { n.s. } \\
\text { n.s. } \\
\text { n.s. } \\
\text { n.s. }\end{array}$ \\
\hline
\end{tabular}

Note: $\mathrm{TCB}=$ target of cyberbullying, $\mathrm{PCB}=$ perpetrator of cyberbullying, $\mathrm{BCB}=$ bystander of cyberbullying.

Source: Own elaboration.

TABLE 4. Differences by place of origin in the QAEU sub-scales.

\begin{tabular}{|l|c|c|c|c|}
\hline \multirow{2}{*}{ QAEU SUB-SCALES } & \multicolumn{2}{|c|}{ ORIGIN } & \multicolumn{2}{|c|}{} \\
\cline { 2 - 5 } & SPANISH & OTHERS & \multicolumn{2}{|c|}{} \\
\cline { 2 - 5 } TCB & $14.46(15.42)$ & $14.56(16.35)$ & -.045 & n.s. \\
\hline \multirow{2}{*}{ PCB } & $7.17(8.92)$ & $7.14(9.37)$ & -.020 & n.s. \\
\hline \multirow{2}{*}{ BCB } & $14.20(17)$ & $16.84(18.98)$ & -1.052 & n.s. \\
\hline
\end{tabular}

Note: $\mathrm{TCB}=$ target of cyberbullying, $\mathrm{PCB}=$ perpetrator of cyberbullying, $\mathrm{BCB}=$ bystander of cyberbullying.

Source: Own elaboration. 
TABLE 5. Differences by level of studies in the QAEU sub-scales.

\begin{tabular}{|c|c|c|c|}
\hline \multirow{2}{*}{ QAEU SUB-SCALES } & \multirow{2}{*}{$\begin{array}{c}\text { LEVEL OF STUDIES } \\
F\end{array}$} & \multirow[b]{2}{*}{ Mean differences } & \multirow[b]{2}{*}{$p$} \\
\hline & & & \\
\hline TCB & $\begin{array}{l}\text { Brown-Forsythe } \\
F(2 ; 24.544)=21.645 \\
p=.000\end{array}$ & $\begin{array}{l}\text { Undergraduate- } \\
\text { Master's }=8.37 \\
\text { Undergraduate- } \\
\text { Other }=-8.67 \\
\text { Master's-Other =-17.04 }\end{array}$ & $\begin{array}{l}.000^{*} \\
\text { n.s. } \\
.07^{*}\end{array}$ \\
\hline PCB & $\begin{array}{l}\text { Brown-Forsythe } \\
F(2 ; 22.451)=9.085 \\
p=.001\end{array}$ & $\begin{array}{l}\text { Grado- Máster }=3.45 \\
\text { Undergraduate- } \\
\text { Other }=-2.30 \\
\text { Master's-Other }=5.76\end{array}$ & $\begin{array}{l}.000^{*} \\
\text { n.s. } \\
.07^{*}\end{array}$ \\
\hline BCB & $\begin{array}{l}\text { Brown-Forsythe } \\
F(2 ; 17.985)=9.764 \\
p=.001\end{array}$ & $\begin{array}{l}\text { Master's-Other }=5.76 \\
\text { Undergraduate- } \\
\text { Master's }=7.14 \\
\text { Undergraduate- } \\
\text { Other }=-5.80 \\
\text { Master's-Other }=-12.93\end{array}$ & $\begin{array}{l}\text { n.s. } \\
.000^{*} \\
\text { n.s. } \\
\text { n.s. }\end{array}$ \\
\hline
\end{tabular}

Note: TCB $=$ target of cyberbullying, $\mathrm{PCB}=$ perpetrator of cyberbullying, $\mathrm{BCB}=$ bystander of cyberbullying.

Source: Own elaboration.

TABLE 6. Differences by faculty in the QAEU sub-scales.

\begin{tabular}{|c|c|c|c|}
\hline \multirow{2}{*}{ QAEU SUB-SCALES } & FACULTY & \multirow[b]{2}{*}{ Mean differences } & \multirow[b]{2}{*}{$p$} \\
\hline & $F$ & & \\
\hline TCB & $\begin{array}{l}\text { Brown-Forsythe } \\
F(3 ; 508.722)=8.240 \\
p=.000\end{array}$ & $\begin{array}{l}\text { Humn. - Soc. Sci. }=1.81 \\
\text { Humn. - Health Sci. }=-.424 \\
\text { Humn. - Sci. }=8.85 \\
\text { Soc. Sci. - Health Sci. }=-2.23 \\
\text { Soc. Sci. - Sci. }=7.04 \\
\text { Health Sci. - Sci. }=9.27\end{array}$ & $\begin{array}{l}\text { n.s. } \\
\text { n.s. } \\
.001^{*} \\
\text { n.s. } \\
.002^{*} \\
.000^{*}\end{array}$ \\
\hline PCB & $\begin{array}{l}\text { Brown-Forsythe } \\
F(3 ; 523.363)=12.136 \\
p=.000\end{array}$ & $\begin{array}{l}\text { Humn. - Soc. Sci. }=2.1 \\
\text { Humn. - Health Sci. }=.56 \\
\text { Humn. - Sci. }=6.5 \\
\text { Soc. Sci. - Health Sci. }=-1.54 \\
\text { Soc. Sci. - Sci. }=4.41 \\
\text { Health Sci. - Sci. }=5.95\end{array}$ & $\begin{array}{l}. \text { n.s. } \\
\text { n.s. } \\
.000 * \\
\text { n.s. } \\
.000 * \\
.000 *\end{array}$ \\
\hline BCB & $\begin{array}{l}\text { Brown-Forsythe } \\
F(3 ; 507.807)=10.126 \\
p=.000\end{array}$ & $\begin{array}{l}\text { Humn. - Soc. Sci. }=2.89 \\
\text { Humn. - Health Sci. }=-1.13 \\
\text { Humn. - Sci. }=10.05 \\
\text { Soc. Sci. - Health Sci. = }-4.02 \\
\text { Soc. Sci. - Sci. = 7.17 } \\
\text { Health Sci. - Sci. =11.19 }\end{array}$ & $\begin{array}{l}\text { n.s. } \\
\text { n.s. } \\
.000^{*} \\
.031^{*} \\
.004^{*} \\
.000^{*}\end{array}$ \\
\hline
\end{tabular}

Note: TCB = target of cyberbullying, $\mathrm{PCB}=$ perpetrator of cyberbullying, $\mathrm{BCB}=$ bystander of cyberbullying.

Source: Own elaboration. 


\section{Discussion}

University students, like people at other educational levels, are vulnerable to bullying by peers (Crosslin et al., 2014; García-Peña et al., 2013). Bystander stood out among the roles involved, followed by aggressors and victims (Hoyos et al., 2009; Paredes et al., 2010; Trujillo et al., 2016). This suggests that situations of aggression are hidden by university students and, therefore, are difficult to detect. Since students from undergraduate degrees, Master's and other courses do not usually interact with all the other students in the university environment and peer relationships are usually reduced to a peer group, situations of aggression can be identified by the closest students (Hoyos et al., 2009). The perception that intimidation is something legitimized within students' social groups means it is viewed positively (Paredes et al., 2010). Therefore, cyberbullying tends to be hidden (Hernández et al., 2007) since the passivity of the victim and bystanders entails subjection to a power that restrains the right to freedom (Torres-Mora, 2010). Cyberbullying may result in negative legal and psychological consequences for the students involved (Álvarez-García, Barreiro-Collazo, et al., 2016; Cassidy et al., 2017, Crosslin et al., 2014; Walker, 2015; Yubero et al., 2017).

In this study, men and women were actors in the different forms of abuse (Anguiano-Carrasco et al., 2011; Faucher et al., 2014; Hoyos et al., 2009; Kokkinos et al., 2014). Women obtained higher values in all of the sub-scales (as perpetrator, target, and bystander), a result that agrees with previous studies (Hoyos et al., 2009; Hoyos et al., 2012). With reference to age, it is important to highlight that the results showed that participants aged under 20 obtained higher values in all the different sub-scales than those in the 20-24 age range. It is noteworthy that in the PCB sub-scale (perpetrator), students aged 30 or more obtained higher values than those in the 20-24 age range, something that does not happen in other age ranges. These data partially coincide with previous studies since a greater presence of intimidation surfaces at the start of studies; this appears to be due to the difficulty of group dynamics and students' participation in them (Hoyos et al., 2009; Hoyos et al., 2012). There were no mean differences in any of the sub-scales relating to the students' origins.

In relation to the level of studies in which the students were enrolled, it should be noted that undergraduate degree students obtained higher values than Master's students in the three sub-scales. On the other hand, in the TCB sub-scale (target), students enrolled in other types of education obtained higher values than Master's students. As with age, the level of studies may be connected to the fact students are starting undergraduate degree studies or different types of education, owing to the difficulty of forming group dynamics (Hoyos et al., 2009; Hoyos et al., 2012).

With regards to the faculty the studies belonged to, the results showed that students in Humanities, Social Sciences, and Health Sciences obtained higher values when compared to students in Scienc- 
es in all the sub-scales. Likewise, Health Sciences students obtained higher values than Social Sciences students in the BCB sub-scale. Previous studies did not give clear proof of the existence of differences relating to faculty, which may be because they only included one or two types of faculty (Molero et al., 2014; Paredes et al., 2010; Yubero et al., 2017).

A relationship between having been the target, perpetrator, and bystander of cyberbullying was demonstrated in predicting TCB, PCB and BCB. The importance of belonging to a particular faculty must be taken into account for PCB and TCB in relation to the level of studies. Our study has allowed a wider vision of intimidation in the university context since it focused on analysing the type of faculty (Humanities, Social Sciences and Education, Health Sciences, and Sciences) as well as the level of studies (undergraduate degree, Master's, or others).

\section{Conclusion}

In conclusion, knowing the origin of school bullying, it is possible to contextualise it in the university community and then promote prevention and intervention programmes. It should be highlighted that these are not alarming situations, but they are worrying because of the incidents found (Hoyos et al., 2009) and the emotional impact (Walker, 2015). Preventive and intervention measures, which allow an analysis of how the issue affects the academic process and interpersonal relationships in the different educational fields, must be implemented (García-Peña et al., 2013; My- ers \& Cowie, 2017). With this objective in mind, it is necessary to promote awareness campaigns and reinforce emotional education as well as the acquisition of social skills (Sastre, 2016) and the values of coexistence (Del Rey et al., 2012), minimise tolerance towards different forms of aggression, diffusion of responsibility and so on (Hoyos et al., 2012), generate strategies to avoid aggression, and promote prosocial behaviour, empathy, and emotional control (Garaigordobil, 2015; Gómez-Ortiz, Romera-Félix, \& Ortega-Ruiz, 2017; Trujillo et al., 2016). It is also necessary to educate students at all educational levels about the safe use of ICT, emphasising the moral and ethical principles against violence (Ortega-Ruiz \& Zych, 2016). Del Rey, Casas, et al. (2012) highlight the important role of the educational institution in relation to technological competence, which means that this competence should be articulated alongside personal autonomy and learning to learn. Therefore, it is essential to encourage cybersocialisation with programmes such as Red (Del Rey, Casas, et al., 2012) or Ciberprogram 2.0 (Garaigordobil \& Martínez-Valderrey, 2014). It is also necessary to promote students' inclusion, coexistence, and cybercoexistence (Garaigordobil, 2015; Llorent et al., 2016), even through (Álvarez-Bermejo, Belmonte-Ureña, Martos-Martínez, Barragán-Martín, \& Simón-Márquez, 2016). What is more, parental supervision of Internet access is also needed as this encourages safe emotional bonds (Bernal, \& Angulo, 2013; Garaigordobil, 2015). Therefore, the university community must assume the importance of promoting coexistence and well-being at university 
(Cassidy et al., 2017) as well as providing legal information for the victims (Myers \& Cowie, 2017). Moreover, the involvement of the whole educational community is essential.

Thinking ahead, it would be relevant to carry out longitudinal studies and include other meaningful variables such as: university students' performance and their involvement in aggressive behaviour (Molero et al., 2014); analysis of whether there is bullying of teaching staff including the school and family climate (López, 2017) or by teachers on students; investigation of harassment within couple relationships (Duran \& Martínez-Pecino, 2015) and in the LGBT community (Walker, 2015); analysis of its appearance along with other associated forms of violent behaviour (Ortega-Barón et al., 2017); investigation of the role of victims of bullying as it has been shown that they also exist in university contexts (Trujillo et al., 2016); investigation of teaching staff's point of view (Cassidy et al., 2017); and investigation of the role of teaching practices in preventing violence between peers (Valdés-Cuervo, Martínez-Ferrer, \& Carlos-Martínez, 2018).

Among the constraints of this study, its transversal character should be noted. Moreover, we should also note the use of self-reporting methods, which may be influenced by social desirability. Finally, since the explained variance percentage is not particularly high, it would be desirable to use other assessment instruments at the same time which would allow identification of other influential variables.

\section{References}

Álvarez-Bermejo, J. A., Belmonte-Ureña, L. J., Martos-Martínez, A., Barragán-Martín, A. B., \& Simón-Márquez, M. D. M (2016). System to Detect Racial-Based Bullying through Gamification. Frontiers Psychology, 7 (1791). doi: https://doi.org/10.3389/fpsyg.2016.01791

Álvarez-García, D., Barreiro-Collazo, A., Núñez, J. C., \& Dobarro, A. (2016). Validity and reliability of the cyber-aggression questionnaire for adolescents (CYBA). The European Journal of Psychology Applied to Legal Context, 8 (2), 69-77. doi: https://doi.org/10.1016/j.ejpal.2016.02.003

Álvarez-García, D., Núñez, J. C., Dobarro, A., \& Rodríguez, C. (2016). Risk factors associated with cybervictimization in adolescence. International Journal of Clinical and Health Psychology, 15, 226-235.

Anguiano-Carrasco, C., \& Vigil-Colet A. (2011). Assessing indirect aggression in aggressors and targets: Spanish adaptation of the Indirect Aggression Scales. Psicothema, 23 (1), 146-152.

Arnaiz, P., Cerezo, F., Giménez A. M., \& Maquilón, J. J. (2016). Conductas de ciberadicción y experiencias de cyberbullying entre adolescentes. Anales de Psicología, 32 (3), 761-769. doi: https://doi.org/10.6018/analesps.32.3.217461

Bernal, C., \& Angulo, F. (2013). Interacciones de los jóvenes andaluces en las redes sociales. $\mathrm{Co}$ municar. Revista Científica de Comunicación y Educación, 20 (40), 25-30. doi: https://doi. org/10.3916/C40-2013-02-02

Buelga, S., Cava, M. J., \& Musitu, G. (2010). Cyberbullying: victimización entre los adolescentes a través del teléfono móvil y de Internet. Psicothema, 22 (4), 784-789.

Cassidy, W., Faucher, C., \& Jackson, M. (2017). Adversity in university: Cyberbullying and its impacts on students, faculty and administrators. International Journal of Environmental Research and Public Health, 14 (8), 888. doi: https://doi.org/10.3390/ijerph14080888

Cerezo, F., Martín, C., Martínez, J. P., Méndez, I., $\&$ Ruiz, C. (2016). Diseño y puesta en marcha del Cuestionario sobre Acoso entre Estudiantes Universitarios (QAUE). Communication presented at the VII International Congress of Psychology and Education. Alicante, España. 
Cohen, J. (1988). Statistical Power Analysis for the Behavioral Sciences. Hillsdale, NJ: Lawrence Erlbaum.

Crosslin, K., \& Golman, K. (2014). "Maybe you don't want to face it". College students' perspectives on cyberbullying. Computers in Human Behavior, 41, 14-20. doi: https://doi. org/10.1016/j.chb.2014.09.007

Del Rey, R., Casas, J. A., \& Ortega-Ruiz, R. (2012). El programa ConRed, una práctica basada en la evidencia. Comunicar. Revista científica de Educación y Comunicación, 20 (39), 129-138.

Del Rey, R., Elipe, P., \& Ortega-Ruiz, R. (2012). Bullying and cyberbullying: overlapping and predictive value of the co-occurrence. Psicothema, 24 (4), 608-613.

Duran, M., \& Martínez-Pecino, R. (2015). Ciberacoso mediante teléfono móvil e internet en las relaciones de noviazgo entre jóvenes. Comunicar. Revista Científica de Educación y Comunicación, 22 (44), 159-167. doi: 10.3916/C442015-17

Faucher, C., Jackson, M., \& Cassidy, W. (2014). Cyberbullying among university students: Gendered experiences, impacts, and perspectives. Education Research International, 2014 (698545). doi: http://dx.doi. org $/ 10.1155 / 2014 / 698545$

Garaigordobil, M. (2011). Prevalencia y consecuencias del cyberbullying: una revision. International Journal of Psychology and Psychological Therapy, 11 (2), 233-254.

Garaigordobil, M. (2015). Ciberbullying en adolescentes y jóvenes del País Vasco: Cambios con la edad. Anales de Psicología, 31 (3), 1069-1076. doi: https://doi.org/10.6018/ analesps.31.3.179151

Garaigordobil, M., \& Martínez-Valderrey, V. M. (2014). Efecto del Cyberprogram 2.0 sobre la reducción de la victimización y la mejora de la competencia social en la adolescencia. Revista de Psicodidáctica, 19 (2), 289-305. doi: https:/ doi.org/10.1387/RevPsicodidact.10239

García-Peña, J. J., Moncada Ortiz, R. M., \& Quintero Gil, J. (2013). El bullying y el suicidio en el escenario universitario. Revista Colombiana de Ciencias Sociales, 4 (2), 298-310.
Gómez-Ortiz, 0., Romera-Félix, E. M., \& Ortega-Ruiz, R. (2017). Multidimensionalidad de la competencia social: medición del constructo y su relación con los roles del bullying. Revista de Psicodidáctica, 22 (1), 37-44. doi: https://doi. org/10.1016/S1136-1034(17)30042-4

Hernández Prados, M. A., \& Solano Fernández, M. I. (2007). Ciberbullying, un problema de acoso escolar. RIED: Revista Iberoamericana de Estudios a Distancia, 10 (1), 17-36.

Hoyos, O. L. R., Llanos M. M., \& Valega, S. J. (2012). El maltrato entre iguales por abuso de poder en el contexto universitario: incidencia, manifestaciones y estrategias de solución. Universitas Psychologica, 11 (3), 793-802.

Hoyos, O. L. R., Romero, L., Valega, S. J., \& Molinares, C. (2009). El maltrato entre iguales por abuso de poder y exclusión social en estudiantes de una universidad privada de la ciudad de Barranquilla. Pensamiento Psicológico, 6 (13), 109-125.

Kokkinos, C. M., Antoniadou, N., \& Markos, A. (2014). Cyber-bullying: An investigation of the psychological profile of university student participants. Journal of Applied Developmental Psychology, 35, 204-2014. doi: 10.1016/j. appdev.2014.04.001

Llorent, V. J., Ortega-Ruiz, R., \& Zych, I. (2016). Bullying and cyberbullying in minorities: Are they more vulnerable than the majority group? Frontiers in Psychology, 7 (1507). doi: https:// doi.org/10.3389/fpsyg.2016.01507

López, M. G. (2017). Influencia del clima escolar y familiar en el acoso escolar y cibernético de universitarios. Revista Mexicana de Investigación en Psicología, 9 (1), 31-44.

Martínez, J. P., Méndez, I., Ruiz-Esteban, C., \& Cerezo, F. (2018). (Unpublished manuscript). $V a$ lidación y fiabilidad del cuestionario sobre Acoso entre Estudiantes Universitarios (QAEU). Departamento de Psicología Evolutiva y de la Educación, Universidad de Murcia, España.

McDougall, L. (1999). A study of bullying in further education. Pastoral Care in Education, 17 (2), 31-37.

Molero, M. M., Gázquez, J. J., Pérez-Fuentes, M. C., \& Soler, F. J. (2014). Rendimiento académico y conducta agresiva en estudiantes universitarios. European Journal of Child Development, Education and Psychopathology, 2 (2), 69-79. 
Myers, C-A., \& Cowie, H. (2017). Bullying at university: The social and legal contexts of cyberbullying among university students. Journal of Cross-Cultural Psychology, 48 (8), 1172-1182. doi: https://doi.org/10.1177/0022022116684208

Olweus, D. (2013). School bullying: development and some important challenges. Annual Review of Clinical Psychology, 9, 751-80. doi:10.1146/ annurev-clinpsy-050212-185516

Ortega-Barón, J., Buelga, S., Cava, M. J., \& Torralba, E. (2017). Violencia escolar y actitud hacia la autoridad de estudiantes agresores de ciberbullying. Revista de Psicodidáctica, 22 (1), 23-28. doi: https://doi.org/10.1016/S11361034(17)30040-0

Ortega-Ruiz, R. (2015). Convivencia y ciberconvivencia. Un modelo educativo para la prevención del acoso y el ciberacoso escolar. Madrid: Machado Libros.

Ortega-Ruiz, R., \& Zych, I. (2016). La ciberconducta y la psicología educativa: retos y riesgos. Psicología Educativa 22, 1-4. doi: https://doi. org/10.1016/j.pse.2016.04.001

Paredes, 0., Sanabria-Ferrand, P. A., González-Quevedo, L. A., \& Moreno Rehalpe, S. P. (2010). "Bullying" en las facultades de Medicina colombianas, mito o realidad. Revista Med, 18 (2), 161172. doi: https://doi.org/10.18359/rmed.1309

Sastre, A. (Coord.) (2016). Yo a eso no juego. Bullying y ciberbullying en la infancia. Madrid: Save the Children España. Retrieved from http://www.observatoriodelainfancia.es/oia/ esp/descargar.aspx?id=4883\&tipo=documento (Consulted on 03/04/2019).

Torres Mora, M. T. (2010). El problema de la violencia entre universitarios abordado desde el enfoque de la Investigación-Participación-Acción. Investigación Universitaria Multidisciplinaria, 9 (9), 27-36.

Trujillo, J. J., \& Romero-Acosta, K. (2016). Variables que evidencian el bullying en un contexto universitario. Revista Encuentros, Universidad Autónoma del Caribe, 14 (01), 41- 54. doi: http://dx.doi.org/10.15665/re.v14i1.668

Valdés-Cuervo, A. A., Martínez-Ferrer, B., \& Carlos-Martínez, E. A. (2018). El rol de las prácticas docentes en la prevención de la violencia escolar entre pares. Revista de Psicodidáctica, 23 (1), 33-38. doi: https://doi.org/10.1016/j. psicod.2017.05.006
Walker, C. (2015). An analysis of cyberbullying among sexual minority university students. Journal of Higher Education Theory and Practice, 15 (7), 44-50.

Yubero, S., Navarro, R., Elche M., Larrañaga, E., \& Ovejero A. (2017). Cyberbullying victimization in higher education: An exploratory analysis of its association with social and emotional factors among Spanish students. Computers in Human Behavior. doi: https://doi.org/10.1016/j. chb.2017.05.037

Zych, I., Ortega-Ruiz, R., \& Marín-López, I. (2016). Cyberbullying: a systematic review of research, its prevalence and assessment issues in Spanish studies. Psicología Educativa, 22, 5-18. doi: https://doi.org/10.1016/j.pse.2016.03.002

\section{Authors' biographies}

Inmaculada Méndez Mateo holds a degree and a PhD in Psychology from the Universidad de Murcia. She works as Assistant Professor at this university and as Quality Coordinator at ISEN Centro Universitario. Her research areas include: social and health risk behaviours in childhood, adolescence and youth (drug consumption, bullying, etc.), as well as active and healthy ageing.

(D) https://orcid.org/0000-0001-8458-5314

Cecilia Ruiz Esteban holds a degree in Educational Sciences from the Universidad Complutense de Madrid, a Master of Science in Education from Bucknell University and a $\mathrm{PhD}$ in Psychology from the Universidad de Murcia. She Works as a Lecturer at this university and as Lead Researcher in the EIPSED Research Group (Research Team in Educational Psychology) of the Universidad de Murcia. Her main areas of research deal with bullying and cyberbullying among peers, personal 
variables in the teaching-learning process and the quality of higher education.

iD https://orcid.org/0000-0002-5836-331X

Juan Pedro Martínez Ramón holds a degree and a PhD in Psychology from the Universidad de Murcia. Degree in Educational Psychology from the Universidad Nacional de Educación a Distancia (UNED) of Madrid. Lecturer in the Department of Developmental and Educational Psychology at the Universidad de Murcia. His areas of research revolve around school coexistence, teacher stress and care for diversity.

(iD https://orcid.org/0000-0003-1770-6598
Fuensanta Cerezo Ramírez is an Assistant Honorary Lecturer at the Universidad de Murcia. Her research focuses on bullying, teacher training, victimisation at school, the perception of teachers, students and parents regarding school coexistence plans, development of a distance programme to improve coexistence through emotional intelligence, bullying and cyberbullying in university classrooms.

(iD https://orcid.org/0000-0002-3160-9930 
revista española de pedagogía año 77, n 273, mayo-agosto 2019

Spanish Journal of Pedagogy

year 77, n. 273, May-August 2019

\section{Table of Contents}

Sumario

\section{Educational and civic-penal responses to antisocial behavior}

Respuestas educativas y cívicopenales a los comportamientos antisociales

Guest editor: Fernando Gil Cantero

Editor invitado: Fernando Gil Cantero

\section{Fernando Gil Cantero}

Introduction: Educational and civic.penal responses to antisocial behavior

Presentación: Respuestas educativas y cívico-penales a los comportamientos antisociales

David Reyero, Fernando Gil Cantero

Education that limits is education that frees

La educación que limita es la que libera

Maria José Bernuz Beneitez, Esther Fernández Molina The pedagogy of juvenile justice: a child.friendly justice

La pedagogía de la justicia de menores: sobre una justicia adaptada a los menores
Marina Martins, Carolina Carvalho

What do teenagers lie about?

¿En qué mienten los adolescentes?

245
Inmaculada Méndez, Cecilia Ruiz Esteban, Juan Pedro Martinez, Fuensanta Cerezo

Cyberbullying according to sociodemographic and academic characteristics among university students Ciberacoso según características sociodemográficas y académicas en estudiantes universitarios

Irene Montiel, José R. Agustina

Educational challenges of emerging risks in cyberspace: foundations of an appropriate strategy for preventing online child victimization

Retos educativos ante los riesgos emergentes en el ciberespacio: claves para una adecuada prevención de la cibervictimización en menores

Maialen Garmendia Larrañaga, Estefanía Jiménez Iglesias, Nekane Larrañaga Aizpuru

Bullying and cyberbullying: victimisation, harassment, and harm. The need to intervene in the educational centre Bullying y ciberbullying: victimización, acoso y daño.

Necesidad de intervenir en el entorno escolar 


\section{Ana Rosser-Limiñana, Raquel Suriá-Martínez}

School adaptation and behavioural and emotional

problems in minors exposed to gender violence

Adaptación escolar y problemas comportamentales y

emocionales en menores expuestos a violencia de género

\section{Fanny T. Añaños-Bedriñana, Miguel Melendro}

\section{Estefanía, Rocio Raya Miranda}

Young women with protective and judicial measures

and their transit towards prison

Mujeres jóvenes con medidas de protección y judiciales y sus

tránsitos hacia la prisión

\section{Book reviews}

Esteban Bara, F. Ética del profesorado [Ethics of

teaching staff] (Juan García Gutiérrez). García

Amilburu, M., Bernal, A., \& González Martín, M.

313

R. Antropología de la educación. La especie educable [Anthropology of education. The educatable species] (Yaiza Sánchez Pérez). Rose, D., \& Martin, J. R. Leer para aprender. Lectura y escritura en las áreas del currículo [Learning to write/reading to learn: Genre knowledge and pedagogy in the SydneySchool: scaffolding democracy in Literacy Classrooms] (Francisco Lorenzo Bergillos). Buxarrais, M. R., \& Vilafranca, I. (Coords.). Una mirada femenina de la educación moral [A feminine view of moral education] (Eric Ortega González).

351

This is the English version of the research articles and book reviews published originally in the Spanish printed version of issue 273 of the revista española de pedagogía. The full Spanish version of this issue can also be found on the journal's website http://revistadepedagogia.org.

ISSN: 0034-9461 (Print), 2174-0909 (Online)

https://revistadepedagogia.org/

Depósito legal: M. 6.020 - 1958

INDUSTRIA GRÁFICA ANZOS, S.L. Fuenlabrada - Madrid 